\title{
Boundary Effect on Asymptotic Behavior of Solutions to the $p$-System with Time-Dependent Damping
}

\author{
Ran Duan $\mathbb{D}^{1},{ }^{1}$ Mina Jiang $\mathbb{D}^{1},{ }^{1}$ and Yinghui Zhang $\mathbb{D}^{2}$ \\ ${ }^{1}$ School of Mathematics and Statistics, Hubei Key Laboratory of Mathematical Sciences, Central China Normal University, \\ Wuhan 430079, China \\ ${ }^{2}$ School of Mathematics and Statistics, Guangxi Normal University, Guilin 541004, China
}

Correspondence should be addressed to Mina Jiang; jmn3911@mail.ccnu.edu.cn

Received 29 June 2019; Accepted 17 August 2019; Published 17 January 2020

Academic Editor: Ciprian G. Gal

Copyright ( $) 2020$ Ran Duan et al. This is an open access article distributed under the Creative Commons Attribution License, which permits unrestricted use, distribution, and reproduction in any medium, provided the original work is properly cited.

In this paper, we consider the asymptotic behavior of solutions to the $p$-system with time-dependent damping on the half-line $\mathbb{R}^{+}=(0,+\infty), v_{t}-u_{x}=0, u_{t}+p(v)_{x}=-\left(\alpha /(1+t)^{\lambda}\right) u$ with the Dirichlet boundary condition $\left.u\right|_{x=0}=0$, in particular, including the constant and nonconstant coefficient damping. The initial data $\left(v_{0}, u_{0}\right)(x)$ have the constant state $\left(v_{+}, u_{+}\right)$at $x=+\infty$. We prove that the solutions time-asymptotically converge to $\left(v_{+}, 0\right)$ as $t$ tends to infinity. Compared with previous results about the $p$-system with constant coefficient damping, we obtain a general result when the initial perturbation belongs to $H^{3}\left(\mathbb{R}^{+}\right) \times H^{2}\left(\mathbb{R}^{+}\right)$. Our proof is based on the time-weighted energy method.

\section{Introduction}

In this paper, we consider the asymptotic behavior and the convergence rates of solutions to the $p$-system with time-dependent damping

$$
\left\{\begin{array}{l}
v_{t}-u_{x}=0, \\
u_{t}+p(v)_{x}=-\frac{\alpha}{(1+t)^{\lambda}} u, \quad x \in \mathbb{R}^{+}, t<0,
\end{array}\right.
$$

with the initial data

$$
\begin{aligned}
& (v(x, 0), u(x, 0))=\left(v_{0}(x), u_{0}(x)\right) \rightarrow \\
& \left(v_{+}, u_{+}\right), \quad v_{+}>0, \quad \text { as } \quad x \rightarrow+\infty,
\end{aligned}
$$

and with the Dirichlet boundary condition

$$
\left.u\right|_{x=0}=0 .
$$

Here, $v>0$ is the specific volume, $u$ is the velocity, the pressure $p(v)$ is a smooth function of $v$ such that $p(v)>0, p^{\prime}(v)<0$, the external term $-\left(\alpha /(1+t)^{\lambda}\right) u$ with physical coefficients $\alpha>0$ and $\lambda \geq 0$, is called a time-dependent damping. $v_{+}>0$ and $u_{+}$are constant states.

For $\alpha=0$, the system (1) reduces to the standard compressible Euler equations. There have been many important developments.
For $\alpha>0, \lambda=0$, the system (1) becomes the compressible Euler equations with damping which model the compressible flow through porous media. There is a huge literature on the investigations of global existence and large time behaviors of smooth solutions to compressible Euler equations with damping. For the Cauchy problem, the global existence of smooth solutions with small initial data has been studied by many authors, cf. [1-4], and the large time behavior of the solutions was carried out by Hsiao and Liu in $[5,6]$ firstly. They showed that the solutions of the Cauchy problem to (1) time-asymptotically behave as those of the following system

$$
\left\{\begin{array}{l}
\bar{v}_{t}-\bar{u}_{x}=0, \\
p(\bar{v})_{x}=-\alpha \bar{u}
\end{array}\right.
$$

or

$$
\left\{\begin{array}{l}
\bar{v}_{t}=-\frac{1}{\alpha} p(\bar{v})_{x x}, \\
\bar{u}=-\frac{1}{\alpha} p(\bar{v})_{x}
\end{array}\right.
$$

with the same end states as $v_{0}(x)$ :

$$
\bar{v}( \pm \infty, t)=v_{ \pm} .
$$

Here, the well-known porous media equation is obtained by Darcy's law, and a better convergence rate and the optimal 
convergence rate when $v(+\infty, 0)=v(-\infty, 0)$ were obtained by Nishihara in $[7,8]$. For the other related results, we refer to [9-11]. Compared with other results, Zhao in [10] got the asymptotic behavior, and convergence rate lay in the facts that the nonlinear diffusion wave $(\bar{v}(x, t), \bar{u}(x, t))$ which satisfies $(4)$ and (6) need not to be weak, and the initial data can be large properly. For the results such as the generalized Riemann problem for a class of quasilinear hyperbolic systems and nonlinear hyperbolic systems of conservation laws with small BV initial data, we refer to $[12,13]$. For other results such as the $p$-system with linear and nonlinear damping, we refer to [14-22].

For the initial-boundary value problems on $\mathbb{R}^{+}$to the equations of viscous conservation laws have been investigated by several authors, cf. [23-27]. For the initial-boundary value problems on $\mathbb{R}^{+}$to the $p$-system with linear damping, see [28, 29], Nishihara and Yang in [29] considered (1)-(3) with $\alpha>0, \lambda=0$, and they got the asymptotic behavior and the convergence rates by perturbing the initial value around the linear diffusion waves $(\bar{v}, \bar{u})(x, t)$ which satisfies

$$
\left\{\begin{array}{l}
\tilde{v}_{t}-\tilde{u}_{x}=0, \quad x \in \mathbb{R}^{+}, \quad t<0, \\
p^{\prime}\left(v_{+}\right) \tilde{v}_{x}=-\alpha \tilde{u}, \\
\left.\tilde{u}\right|_{x=0}=0,\left.\quad(\tilde{v}, \tilde{u})\right|_{x=\infty}=\left(v_{+}, 0\right) .
\end{array}\right.
$$

For $\alpha>0, \lambda>0$, the system (1) reduces to compressible Euler equations with time-dependent damping. Hou et al. [30] considered the global existence of smooth solutions for $0 \leq \lambda \leq 1$ in three space dimensions; they proved that the solutions will blow up in finite time for $\lambda \geq 1$. In [31, 32], Pan obtained the existence and decay rates of solutions near constant states $(1,0)$ for $\lambda=1$. For the Cauchy problem of the system (1), Cui et al. in [33] proved that the solutions time-asymptotically converge to the diffusion wave whose profile is self-similar solution to the corresponding parabolic equation. For other results, we refer to [34].

However, to our knowledge, there are few results for the initial-boundary value problem of the system (1). In this paper, we obtained the asymptotic behavior and the decay rates for the solutions of the system (1) with initial-boundary value data. Because the time-dependent damping will lead to some new phenomena and severe mathematical difficulties, we will use some new techniques here. As usual, we want to get the convergence rates of the solution by the usual energy estimates and some elementary computations. However, the time-dependent damping brings some extra terms, such as in the right hand of (4.19) and (4.61), $C \int(1+t)^{-\kappa} V^{2} d x$, $C \int(1+t)^{\beta+\lambda-1}\left(V_{x}^{2}+V_{t}^{2}\right) d x, \quad C \int(1+t)^{\beta+2 \lambda}\left(V_{x x}^{2}+V_{x t}^{2}\right) d x$. To deal with these terms, we divide the time interval into two regions, $\left[0, T_{0}\right)$ and $\left[T_{0}, \infty\right)$. In the bounded region $\left[0, T_{0}\right)$, we obtain the desired estimates by using Gronwall's inequality and the usual energy estimates. In unbounded region $\left[T_{0}, \infty\right)$, we observe the bad terms could be bounded by the corresponding energy functional when $t \geq T_{0}$. By using the Gronwall's inequality and the time-weighted energy method, we also obtain the desired decay estimates, and the desired decay rates will not be lost by using the weight function $(1+t)^{\beta}$.

The rest of this paper is organized as follows. In Section 2, we reformulate the problem (1)-(3) and state the main theorems. In Section 3, a priori estimates of finite time intervals will be given. In Section 4, decay rates will be given.

1.1. Notation. Hereafter, we denote several generic positive constants depending on $T$ by $C(T), C$ or $O(1)$ denote the generic positive constants depending only on the initial data and the physical coefficients $\alpha, \lambda$, but independent of the time without any confusion. $\varepsilon$ will always be used to represent sufficiently small positive constants. $L^{p}=L^{p}\left(\mathbb{R}^{+}\right)(1 \leq p \leq \infty)$ denotes usual Lebesgue space with the norm

$$
\begin{gathered}
\|f\|_{L^{p}}=\left(\int_{\mathbb{R}^{+}}|f(x)|^{p} d x\right)^{1 / p}, \quad 1 \leq p<\infty, \\
\|f\|_{L^{\infty}}=\sup _{\mathbb{R}^{+}}|f(x)|,
\end{gathered}
$$

and the integral region $\mathbb{R}^{+}$will be omitted without any confusion. $H^{l}(l \geq 0)$ denotes the usual $l$ th-order Sobolev space with the norm

$$
\|f\|_{l}=\left(\sum_{j=0}^{l}\left\|\partial_{x}^{j} f\right\|^{2}\right)^{1 / 2}
$$

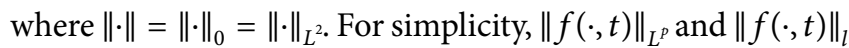
are denoted by $\|f(t)\|_{L^{p}}$ and $\|f(t)\|_{l}$ respectively.

\section{Main Theorems}

From asymptotic analysis, it is well known that the first term $u_{t}$ of $(1)_{2}$ decay to zero, as $t \rightarrow \infty$, faster than others. Expecting $(v, u)(x, t) \rightarrow\left(v_{+}, 0\right), t \rightarrow \infty$ we approximate this by the solution $(\bar{v}(x, t), \bar{u}(x, t))$ of

$$
\left\{\begin{array}{l}
\bar{v}_{t}-\bar{u}_{x}=0, \\
p^{\prime}\left(v_{+}\right) \bar{v}_{x}=-\frac{\alpha}{(1+t)^{\lambda}} \bar{u}, \quad x \in \mathbb{R}^{+}, \quad t<0, \\
(\bar{v}(x, t), \bar{u}(x, t)) \rightarrow\left(v_{+}, 0\right), \quad v_{+}<0, \quad \text { as } x \rightarrow+\infty \\
\left.\bar{u}\right|_{x=0}=0\left(\text { or }\left.\bar{v}_{x}\right|_{x=0}=0\right),
\end{array}\right.
$$

Explicitly,

$$
\bar{v}(x, t)=v_{+}+\frac{\delta_{0}}{\sqrt{4 \kappa \pi(1+t)^{\lambda+1}}} e^{-\left(\left((\lambda+1) x^{2}\right) /\left(4 \kappa(1+t)^{\lambda+1}\right)\right)},
$$

where $\kappa:=\left(-p^{\prime}\left(v_{+}\right) / \alpha\right)>0$ and $\delta_{0}$ is defined by

$$
\delta_{0}=2 \sqrt{\lambda+1}\left(\int_{0}^{+\infty}\left(v_{0}(x)-v_{+}\right) d x-B(0) u_{+}\right)
$$

$B(0)$ is to be defined later.

Note that $\bar{u}(\infty, t)=0$. Hinted by (1), we suppose

$$
u(x, t) \rightarrow \beta(t) u_{+}, \text {as } x \rightarrow+\infty,
$$

where

$$
\beta(t)= \begin{cases}e^{-(\alpha / 1-\lambda)\left[(1+t)^{1-\lambda}-1\right]}, & \text { if } \lambda \in[0,1], \\ (1+t)^{-\alpha}, & \text { if } \lambda=1 .\end{cases}
$$

We define the auxiliary function $(\widehat{v}, \widehat{u})(x, t)$ by

$$
\left\{\begin{array}{l}
\widehat{v}(x, t)=B(t) m_{0}(x) u_{+}, \\
\widehat{u}(x, t)=u_{+} \beta(t) \int_{0}^{x} m_{0}(y) d y,
\end{array}\right.
$$


where $B(t)=-\int_{t}^{\infty} \beta(\tau) d \tau$, and $m_{0}(x)$ is a smooth function with compact support such that

$$
\int_{0}^{\infty} m_{0}(y) d y=1, \quad \operatorname{supp} m_{0}(x) \subset \mathbb{R}^{+} .
$$

Then $(\widehat{v}, \widehat{u})(x, t)$ satisfies

$$
\left\{\begin{array}{l}
\widehat{v}_{t}-\widehat{u}_{x}=0, \\
\widehat{u}_{t}=-\frac{\alpha}{(1+t)^{2}} \widehat{u}, \quad x \in \mathbb{R}^{+}, \quad t<0, \\
\left.\widehat{u}\right|_{x=0}=0,\left.(\widehat{v}, \widehat{u})\right|_{x=\infty}=\left(0, u_{+} \beta(t)\right) .
\end{array}\right.
$$

By direct calculation, we have

Lemma 2.1. For $0 \leq \lambda<1$, there exist constants $0<9<1-\lambda, c, C>0$, such that for any $t \geq 0$, we have

$$
\|\widehat{v}\|_{L^{1}}+\|\widehat{v}\|_{L^{\infty}}+\left\|\widehat{v}_{t}\right\|_{L^{\infty}} \leq C\left|u_{+}\right| e^{-c t^{9}}
$$

Lemma 2.2. The solution $\bar{v}(x, t)$ of (10) satisfies the following dissipative estimates

$$
\begin{gathered}
\int\left|\bar{v}_{x}(x, t)\right|^{2} d x \leq C\left|\delta_{0}\right|^{2}(1+t)^{-(3(\lambda+1) / 2)} \\
\int\left|\bar{v}_{t}(x, t)\right|^{2} d x \leq C\left|\delta_{0}\right|^{2}(1+t)^{-(\lambda / 2)-(5 / 2)} \\
\int\left|\bar{v}_{x x}(x, t)\right|^{2} d x \leq C\left|\delta_{0}\right|^{2}(1+t)^{-(5(\lambda+1) / 2)} \\
\int\left|\bar{v}_{x t}(x, t)\right|^{2} d x \leq C\left|\delta_{0}\right|^{2}(1+t)^{-(3 \lambda / 2)-(7 / 2)} \\
\int\left|\bar{v}_{x x x}(x, t)\right|^{2} d x \leq C\left|\delta_{0}\right|^{2}(1+t)^{-(7(\lambda+1) / 2)} \\
\int\left|\bar{v}_{x x t}(x, t)\right|^{2} d x \leq C\left|\delta_{0}\right|^{2}(1+t)^{-(5 \lambda / 2)-(9 / 2)} \\
\int\left|\bar{v}_{t t}(x, t)\right|^{2} d x \leq C\left|\delta_{0}\right|^{2}(1+t)^{-(\lambda / 2)-(9 / 2)} \\
\int\left|\left(p^{\prime}\left(v_{+}\right)-p^{\prime}(\bar{v})\right) \bar{v}_{x}(x, t)\right|^{2} d x \leq C\left|\delta_{0}\right|^{2}(1+t)^{-(5(\lambda+1) / 2)} \\
\left.\left.\int p^{\prime}\left(v_{+}\right)-p^{\prime}(\bar{v})\right) \bar{v}_{x}(x, t)\right)\left._{x}\right|^{2} d x \leq C\left|\delta_{0}\right|^{2}(1+t)^{-(7(\lambda+1) / 2)}
\end{gathered}
$$

Corollary 2.3. The solution $\bar{v}(x, t)$ of (10) satisfies the following dissipative estimates

$$
\begin{gathered}
\left\|\bar{v}_{x}(\cdot, t)\right\|_{L^{\infty}} \leq C\left|\delta_{0}\right|(1+t)^{-(\lambda+1)}, \\
\left\|\bar{v}_{t}(\cdot, t)\right\|_{L^{\infty}} \leq C\left|\delta_{0}\right|(1+t)^{-(\lambda / 2)-(3 / 2)}, \\
\left\|\bar{v}_{x x}(\cdot, t)\right\|_{L^{\infty}} \leq C\left|\delta_{0}\right|(1+t)^{-(3(\lambda+1) / 2)}, \\
\left\|\bar{v}_{x t}(\cdot, t)\right\|_{L^{\infty}} \leq C\left|\delta_{0}\right|(1+t)^{-\lambda-2} .
\end{gathered}
$$

Combining (1) with (10) and (17), we have

$$
\left\{\begin{array}{l}
(v-\bar{v}-\widehat{v})_{t}-(u-\bar{u}-\widehat{u})_{x}=0, \\
(u-\bar{u}-\widehat{u})_{t}+(p(v)-p(\bar{v}))_{x}=-\frac{\alpha}{(1+t)^{\lambda}} \\
\cdot(u-\bar{u}-\widehat{u})-\bar{u}_{t}+\left(p^{\prime}\left(v_{+}\right)-p^{\prime}(\bar{v})\right) \bar{v}_{x} .
\end{array}\right.
$$

By virtue of (32) and (12),

$\int_{0}^{+\infty}(v-\bar{v}-\widehat{v})(y, t) d y=\int_{0}^{+\infty}\left(v_{0}(x)-v_{+}\right) d x-B(0) u_{+}-\frac{\delta_{0}}{2 \sqrt{1+\lambda}}=0$,

and hence we reach the setting of perturbation

$$
\left\{\begin{array}{l}
V(x, t):=-\int_{x}^{\infty}(v-\bar{v}-\widehat{v})(y, t) d y, \\
U(x, t):=u(x, t)-\bar{u}(x, t)-\widehat{u}(x, t) .
\end{array}\right.
$$

From (32) and (34), we deduce that $(V, U)(x, t)$ solves the following problem

$$
\left\{\begin{array}{l}
V_{t}-U=0, \quad x \in \mathbb{R}^{+}, \quad t<0, \\
U_{t}+\left(p\left(V_{x}+\bar{v}+\widehat{v}\right)-p(\bar{v})\right)_{x}+\frac{\alpha}{(1+t)^{\lambda}} U=-\bar{u}_{t}+\left(p^{\prime}\left(v_{+}\right)-p^{\prime}(\bar{v})\right) \bar{v}_{x},
\end{array}\right.
$$

with the initial data

$$
\begin{aligned}
(V, U)(x, 0)= & \left(V_{0}, U_{0}\right)(x):=\left(-\int_{x}^{\infty}\left(v_{0}(y)-\bar{v}(y, 0)-\widehat{v}(y, 0)\right) d y,\right. \\
& \left.u_{0}(x)-\bar{u}(x, 0)-\widehat{u}(x, 0)\right),
\end{aligned}
$$

and the boundary condition

$$
\left.U\right|_{x=0}=0,
$$

or the linearized problem around $\bar{v}$

$$
\left\{\begin{array}{l}
V_{t}-U=0, \quad x \in \mathbb{R}^{+}, \quad t>0 \\
U_{t}+\left(p^{\prime}(\bar{v}) V_{x}\right)_{x}+\frac{\alpha}{(1+t)^{\lambda}} U=F
\end{array}\right.
$$

where

$$
\begin{aligned}
F= & \frac{(1+t)^{\lambda}}{\alpha} p^{\prime}\left(v_{+}\right) \bar{v}_{x t}+\frac{\lambda(1+t)^{\lambda-1}}{\alpha} p^{\prime}\left(v_{+}\right) \bar{v}_{x} \\
& -\left(p\left(V_{x}+\bar{v}+\widehat{v}\right)-p(\bar{v})-p^{\prime}(\bar{v}) V_{x}\right)_{x}+\left(p^{\prime}\left(v_{+}\right)-p^{\prime}(\bar{v})\right) \bar{v}_{x} .
\end{aligned}
$$


In this paper, we do not consider the case $\lambda=1$. For the case of $0 \leq \lambda<1$, from (12), we have

$$
\left|\delta_{0}\right| \leq C\left(\left\|v_{0}(x)-v_{+}\right\|_{L^{1}}+\left|u_{+}\right|\right),
$$

we obtain the following theorems.

Theorem 2.4 (the case of $0 \leq \lambda<3 / 5$ ). Suppose that $v_{0}(x)-v_{+} \in L^{1},\left(V_{0}, U_{0}\right) \in H^{3} \times H^{2}$, both $\delta=\left\|v_{0}(x)-v_{+}\right\|_{L^{1}}+\left|u_{+}\right|$ and $\left\|V_{0}\right\|_{3}+\left\|U_{0}\right\|_{2}$ are sufficiently small. Then there exists a unique time-global solution $(V, U)(x, t)$ of (35)-(37) which satisfies

$$
\begin{gathered}
V \in C^{i}\left([0, \infty) ; H^{3-i}\right), \quad i=0,1,2,3, \\
U \in C^{i}\left([0, \infty) ; H^{2-i}\right), \quad i=0,1,2,
\end{gathered}
$$

and moreover

$$
\begin{aligned}
& \sum_{k=0}^{3}(1+t)^{(\lambda+1) k}\left\|\partial_{x}^{k} V(t)\right\|^{2}+\sum_{k=0}^{2}(1+t)^{(\lambda+1) k+2}\left\|\partial_{x}^{k} U(t)\right\|^{2} \\
& \quad+\int_{0}^{t}\left(\sum_{j=1}^{3}(1+s)^{(\lambda+1) j-1}\left\|\partial_{x}^{j} V(s)\right\|^{2}+\sum_{j=0}^{2}(1+s)^{(\lambda+1) j+1}\left\|\partial_{x}^{j} U(s)\right\|^{2}\right) d s \\
& \quad \leq O(1)\left(\left\|V_{0}\right\|_{3}^{2}+\left\|U_{0}\right\|_{2}^{2}+\delta\right) .
\end{aligned}
$$

Theorem 2.5 (the case of $3 / 5<\lambda<1$ ). Suppose that $v_{0}(x)-v_{+} \in L^{1},\left(V_{0}, U_{0}\right) \in H^{3} \times H^{2}$, both $\delta=\left\|v_{0}(x)-v_{+}\right\|_{L^{1}}+\left|u_{+}\right|$ and $\left\|V_{0}\right\|_{3}+\left\|U_{0}\right\|_{2}$ are sufficiently small. Then there exists a unique time-global solution $(V, U)(x, t)$ of (35)-(37) which satisfies

$$
\begin{aligned}
& V \in C^{i}\left([0, \infty) ; H^{3-i}\right), \quad i=0,1,2,3, \\
& U \in C^{i}\left([0, \infty) ; H^{2-i}\right), \quad i=0,1,2, \\
& \sum_{k=0}^{3}(1+t)^{(\lambda+1) k+(3 / 2)-(5 \lambda / 2)}\left\|\partial_{x}^{k} V(t)\right\|^{2} \\
& \quad+\sum_{k=0}^{2}(1+t)^{(\lambda+1) k+(7 / 2)-(5 \lambda / 2)}\left\|\partial_{x}^{k} U(t)\right\|^{2} \\
& \leq O(1)\left(\left\|V_{0}\right\|_{3}^{2}+\left\|U_{0}\right\|_{2}^{2}+\delta\right),
\end{aligned}
$$

and for any $\beta \in(3 / 2-3 \lambda / 2, \lambda)$, we have

$$
\begin{aligned}
& \int_{0}^{t}\left(\sum_{j=0}^{3}(1+s)^{(\lambda+1)(j-1)+\beta}\left\|\partial_{x}^{j} V(s)\right\|^{2}\right. \\
& \left.\quad+\sum_{j=0}^{2}(1+s)^{(\lambda+1) j+\beta-\lambda+1}\left\|\partial_{x}^{j} U(s)\right\|^{2}\right) d s \\
& \quad \leq O(1)(1+t)^{\beta+(3 \lambda / 2)-(3 / 2)}\left(\left\|V_{0}\right\|_{3}^{2}+\left\|U_{0}\right\|_{2}^{2}+\delta\right) .
\end{aligned}
$$

Theorem 2.6 (the case of $\lambda=3 / 5)$. Suppose that $v_{0}(x)-v_{+} \in L^{1}$, $\left(V_{0}, U_{0}\right) \in H^{3} \times H^{2}, \quad$ both $\delta=\left\|v_{0}(x)-v_{+}\right\|_{L^{1}}+\left|u_{+}\right|$and $\left\|V_{0}\right\|_{3}+\left\|U_{0}\right\|_{2}$ are sufficiently small. Then there exists a unique time-global solution $(V, U)(x, t)$ of (35)-(37) which satisfies

$$
\begin{gathered}
V \in C^{i}\left([0, \infty) ; H^{3-i}\right), \quad i=0,1,2,3, \\
U \in C^{i}\left([0, \infty) ; H^{2-i}\right), \quad i=0,1,2,
\end{gathered}
$$

furthermore, we have for any sufficiently small $\varepsilon>0$

$$
\begin{aligned}
\sum_{k=0}^{3}(1+t)^{8 k / 5}\left\|\partial_{x}^{k} V(t)\right\|^{2}+\sum_{k=0}^{2}(1+t)^{(8 k / 5)+2}\left\|\partial_{x}^{k} U(t)\right\|^{2} \\
\quad+\int_{0}^{t}\left(\sum_{j=1}^{3}(1+s)^{(8 j / 5)-1}\left\|\partial_{x}^{j} V(s)\right\|^{2}+\sum_{j=0}^{2}(1+s)^{(8 j / 5)+1}\left\|\partial_{x}^{j} U(s)\right\|^{2}\right) d s \\
\leq O(1)(1+t)^{\varepsilon}\left(\left\|V_{0}\right\|_{3}^{2}+\left\|U_{0}\right\|_{2}^{2}+\delta\right) .
\end{aligned}
$$

Remark 1. For the case of $\lambda=0$, the convergence rate shown in (43) is the same as that in Nishihara and Yang in [29]. In other words, our estimates give a general result by the elementary method.

Remark 2. All results are obtained under the condition that any data are small. For large data, the asymptotic behavior of the solutions of (1) with initial data or initial-boundary data will be very difficult, and we will consider them later.

\section{A Priori Estimates on Finite Time Intervals}

Compared with the constant damping, the time-dependent damping may bring some extra terms. To deal with the terms, we divide the time interval into two regions, $[0, T]$ and $[T, \infty]$, then apply the $L^{2}$-energy method and the Gronwall's inequality to obtain the desired estimates. Much of this section is based on the paper [33].

We now devote ourselves to the a priori estimates of the solution $(V, U)(x, t)$ under the a priori assumption

$$
N(T):=\sup _{0<t<T}\left\{\sum_{k=0}^{3}\left\|\partial_{x}^{k} V(t)\right\|^{2}+\sum_{k=0}^{2}\left\|\partial_{x}^{k} U(t)\right\|^{2}\right\} \leq \varepsilon^{2},
$$

where $0<\varepsilon \ll 1$.

By Sobolev inequality and the equation (35), we have

$$
\left\{\begin{array}{l}
\left\|\partial_{x}^{k} V(t)\right\|_{L^{\infty}} \leq \varepsilon, \quad k=0,1,2 \\
\left\|\partial_{x}^{k} V_{t}(t)\right\|_{L^{\infty}} \leq \varepsilon, \quad k=0,1
\end{array}\right.
$$

which will be used later.

Proposition 3.1. Under the conditions of Theorem 2.4 for any given $T>0,0 \leq \lambda<1, \alpha>0$, the solution $\left(V, V_{t}\right)(x, t)$ to the initial-boundary problem (35), (36), (37) on $[0, T]$ satisfying

$$
\|V\|_{1}^{2}+\left\|V_{t}\right\|^{2}+\int_{0}^{t}\left(\left\|V_{x}\right\|^{2}+\left\|V_{t}\right\|^{2}\right) d s \leq C(T)\left(\left\|V_{0}\right\|_{1}^{2}+\left\|U_{0}\right\|^{2}+\delta\right),
$$

$\left\|V_{x x}\right\|^{2}+\left\|V_{x t}\right\|^{2}+\int_{0}^{t}\left(\left\|V_{x x}\right\|^{2}+\left\|V_{x t}\right\|^{2}\right) d s \leq C(T)\left(\left\|V_{0}\right\|_{2}^{2}+\left\|U_{0}\right\|_{1}^{2}+\delta\right)$, 
and

$$
\begin{aligned}
\left\|V_{x x x}\right\|^{2} & +\left\|V_{x x t}\right\|^{2}+\int_{0}^{t}\left(\left\|V_{x x x}\right\|^{2}+\left\|V_{x x t}\right\|^{2}\right) d s \\
& \leq C(T)\left(\left\|V_{0}\right\|_{3}^{2}+\left\|U_{0}\right\|_{2}^{2}+\delta\right) .
\end{aligned}
$$

Since it suffices to establish the estimates for sufficiently smooth solution, the equations in (35), (33) and $\left.U\right|_{x=0}=0$ give the following boundary conditions for higher order derivatives

$$
V(0, t)=V_{x x}(0, t)=V_{t}(0, t)=V_{t x x}(0, t)=0, \text { etc. }
$$

(39) can be rewritten as the problem to the second order wave of $V$

$$
\left\{\begin{array}{l}
V_{t t}+\left(p^{\prime}(\bar{v}) V_{x}\right)_{x}+\frac{\alpha}{(1+t)^{\lambda}} V_{t}=F \\
\left|\left(V, V_{t}\right)\right|_{t=0}=\left(V_{0}, U_{0}\right)(x), \\
|V|_{x=0}=0 .
\end{array}\right.
$$

Step 1. First, multiplying (57) by $V$ and integrating the resulting equation with respect to $x$ over $\mathbb{R}^{+}$, we can get

$$
\begin{aligned}
& \frac{d}{d t} \int\left[V V_{t}+\frac{\alpha}{2}(1+t)^{-\lambda} V^{2}\right] d x+\frac{\alpha \lambda}{2} \int(1+t)^{-\lambda-1} V^{2} d x \\
& \quad-\int p^{\prime}(\bar{v}) V_{x}^{2} d x=\int V_{t}^{2} d x+\int F V d x
\end{aligned}
$$

We now estimate the last term on the right hand of (58), from (39), we have

$$
\begin{aligned}
\int F V d x= & \int\left[\frac{(1+t)^{\lambda}}{\alpha} p^{\prime}\left(v_{+}\right) \bar{v}_{x t}+\frac{\lambda(1+t)^{\lambda-1}}{\alpha} p^{\prime}\left(v_{+}\right) \bar{v}_{x}\right. \\
& \left.-\left(p\left(V_{x}+\bar{v}+\widehat{v}\right)-p(\bar{v})-p^{\prime}(\bar{v}) V_{x}\right)_{x}+\left(p^{\prime}\left(v_{+}\right)-p^{\prime}(\bar{v})\right) \bar{v}_{x}\right] V d x .
\end{aligned}
$$

From Lemma 2.2, the Cauchy-Schwarz's inequality and noticing $p^{\prime}(v) \leq-C_{0}<0$, we have

$$
\begin{aligned}
& \int \frac{(1+t)^{\lambda}}{\alpha} p^{\prime}\left(v_{+}\right) \bar{v}_{x t} V d x=-\int \frac{(1+t)^{\lambda}}{\alpha} p^{\prime}\left(v_{+}\right) \bar{v}_{t} V_{x} d x \\
& \leq \frac{C_{0}}{8} \int V_{x}^{2} d x+C \int(1+t)^{2 \lambda}\left|\bar{v}_{t}\right|^{2} d x \leq \frac{C_{0}}{8} \int V_{x}^{2} d x+C \delta^{2},
\end{aligned}
$$

and

$$
\begin{aligned}
& \int \frac{\lambda(1+t)^{\lambda-1}}{\alpha} p^{\prime}\left(v_{+}\right) \bar{v}_{x} V d x \leq \frac{\alpha \lambda}{8} \int(1+t)^{-\lambda-1} V^{2} d x \\
& +C \int(1+t)^{3 \lambda-1}\left|\bar{v}_{x}\right|^{2} d x \leq \frac{\alpha \lambda}{8} \int(1+t)^{-\lambda-1} V^{2} d x+C \delta^{2} .
\end{aligned}
$$

By using Lemma 2.1, Lemma 2.2 and a priori assumption (52), we have

$$
\begin{aligned}
- & \int\left(p\left(V_{x}+\bar{v}+\widehat{v}\right)-p(\bar{v})-p^{\prime}(\bar{v}) V_{x}\right)_{x} V d x \\
= & \int\left(p(V x+\bar{v}+\widehat{v})-p(\bar{v})-p^{\prime}(\bar{v}) V_{x}\right) V_{x} d x \\
\leq & \frac{C_{0}}{8} \int V_{x}^{2} d x+C \int\left(|\widehat{v}|^{2}+\left|V_{x}\right|^{4}+|\widehat{v}|^{4}\right) d x \\
\leq & \frac{C_{0}}{8} \int V_{x}^{2} d x+C \varepsilon^{2} \int V_{x}^{2} d x+C \delta^{2},
\end{aligned}
$$

and

$$
\begin{aligned}
& \int\left(p^{\prime}\left(v_{+}\right)-p^{\prime}(\bar{v})\right) \bar{v}_{x} V d x \leq \frac{\alpha \lambda}{8} \int(1+t)^{-\lambda-1} V^{2} d x \\
& +C \int\left(\left(p^{\prime}\left(v_{+}\right)-p^{\prime}(\bar{v})\right) \bar{v}_{x}\right)^{2}(1+t)^{\lambda+1} d x \\
& \quad \leq \frac{\alpha \lambda}{8} \int(1+t)^{-\lambda-1} V^{2} d x+C \delta^{2} .
\end{aligned}
$$

Substituting (60)-(63) into (58), and noting the smallness of $\varepsilon$, we have

$$
\begin{aligned}
& \frac{d}{d t} \int\left[V V_{t}+\frac{\alpha}{2}(1+t)^{-\lambda} V^{2}\right] d x+\frac{\alpha \lambda}{4} \int(1+t)^{-\lambda-1} V^{2} d x \\
& \quad+\frac{C_{0}}{4} \int V_{x}^{2} d x \leq \int V_{t}^{2} d x+C \delta^{2} .
\end{aligned}
$$

Next, multiplying (57) by $(1+t)^{\lambda} V_{t}$ and integrating the resulting equation with respect to $x$ over $\mathbb{R}^{+}$and using Lemma 2.2, we can get

$$
\begin{aligned}
& \frac{1}{2} \frac{d}{d t} \int\left[(1+t)^{\lambda} V_{t}^{2}-(1+t)^{\lambda} p^{\prime}(\bar{v}) V_{x}^{2}\right] d x \\
& \quad+\alpha \int V_{t}^{2} d x=-\frac{1}{2} \int(1+t)^{\lambda} p^{\prime \prime}(\bar{v}) \bar{v}_{t} V_{x}^{2} d x \\
& \quad-\frac{\lambda}{2} \int(1+t)^{\lambda-1} p^{\prime}(\bar{v}) V_{x}^{2} d x+\frac{\lambda}{2} \int(1+t)^{\lambda-1} V_{t}^{2} d x \\
& \quad+\int(1+t)^{\lambda} F V_{t} d x \leq C \int(1+t)^{\lambda-1}\left(V_{x}^{2}+V_{t}^{2}\right) d x \\
& \quad+\int(1+t)^{\lambda} F V_{t} d x .
\end{aligned}
$$

Now, we estimate the last term in the right hand of (65) as follows:

$$
\begin{aligned}
\int(1+t)^{\lambda} F V_{t} d x= & \int\left[\frac{(1+t)^{2 \lambda}}{\alpha} p^{\prime}\left(v_{+}\right) \bar{v}_{x t}+\frac{\lambda(1+t)^{2 \lambda-1}}{\alpha} p^{\prime}\left(v_{+}\right) \bar{v}_{x}\right. \\
& -(1+t)^{\lambda}\left(p\left(V_{x}+\bar{v}+\widehat{v}\right)-p(\bar{v})-p^{\prime}(\bar{v}) V_{x}\right)_{x} \\
& \left.+(1+t)^{\lambda}\left(p^{\prime}(v+)-p^{\prime}(\bar{v})\right) \bar{v} x\right] V_{t} d x .
\end{aligned}
$$

By using Lemma 2.1, Lemma 2.2 and a priori assumption (52), we have

$$
\begin{aligned}
& \int \frac{(1+t)^{2 \lambda}}{\alpha} p^{\prime}\left(v_{+}\right) \bar{v}_{x t} V_{t} d x \leq \frac{\alpha}{4} \int V_{t}^{2} d x \\
& +C \int(1+t)^{4 \lambda}\left|\bar{v}_{x t}\right|^{2} d x \leq \frac{\alpha}{4} \int V_{t}^{2} d x+C \delta^{2}, \\
& \int \frac{\lambda(1+t)^{2 \lambda-1}}{\alpha} p^{\prime}\left(v_{+}\right) \bar{v}_{x} V_{t} d x \leq \frac{\alpha}{4} \int V_{t}^{2} d x \\
& +C \int(1+t)^{4 \lambda-2}\left|\bar{v}_{x}\right|^{2} d x \leq \frac{\alpha}{4} \int V_{t}^{2} d x+C \delta^{2},
\end{aligned}
$$




$$
\begin{aligned}
- & \int(1+t)^{\lambda}\left(p\left(V_{x}+\bar{v}+\widehat{v}\right)-p(\bar{v})-p^{\prime}(\bar{v}) V_{x}\right) V_{x} V_{t} d x \\
= & \int(1+t)^{\lambda}\left(p\left(V_{x}+\bar{v}+\widehat{v}\right)-p(\bar{v})-p^{\prime}(\bar{v}) V_{x}\right) V_{x t} d x \\
= & (1+t)^{\lambda} \frac{d}{d t} \int\left(\int_{\bar{v}}^{V_{x}+\bar{v}+\hat{v}} p(s) d s-p(\bar{v}) V_{x}-\frac{1}{2} p^{\prime}(\bar{v}) V_{x}^{2}\right) d x \\
& -\int(1+t)^{\lambda}\left(p\left(V_{x}+\bar{v}+\widehat{v}\right)-p(\bar{v})-p^{\prime}(\bar{v}) V_{x}-\frac{1}{2} p^{\prime \prime}(\bar{v}) V_{x}^{2}\right) \bar{v}_{t} d x \\
& -\int(1+t)^{\lambda} p\left(V_{x}+\bar{v}+\widehat{v}\right) \widehat{v}_{t} d x \\
\leq & \frac{d}{d t} \int(1+t)^{\lambda}\left(\int_{\bar{v}}^{V_{x}+\bar{v}+\widehat{v}} p(s) d s-p(\bar{v}) V_{x}-\frac{1}{2} p^{\prime}(\bar{v}) V_{x}^{2}\right) d x \\
& -\lambda \int(1+t)^{\lambda-1}\left(\int_{\bar{v}}^{V_{x}+\bar{v}+\hat{v}} p(s) d s-p(\bar{v}) V_{x}-\frac{1}{2} p^{\prime}(\bar{v}) V_{x}^{2}\right) d x \\
& +C \delta \int(1+t)^{\lambda-1}\left(|\widehat{v}|+\left|V_{x}\right|^{3}\right) d x+C \int(1+t)^{\lambda}\left|\widehat{v}_{t}\right| d x \\
\leq & \frac{d}{d t} \int(1+t)^{\lambda}\left(\int_{\bar{v}}^{V_{x}+\bar{v}+\widehat{v}} p(s) d s-p(\bar{v}) V_{x}-\frac{1}{2} p^{\prime}(\bar{v}) V_{x}^{2}\right) d x \\
& +C \varepsilon \int(1+t)^{\lambda-1} V_{x}^{2} d x+C \delta^{2},
\end{aligned}
$$

and

$$
\begin{aligned}
& \int(1+t)^{\lambda}\left(p^{\prime}(v+)-p^{\prime}(\bar{v})\right) \bar{v}_{x} V_{t} d x \\
& \quad \leq \frac{\alpha}{4} \int V_{t}^{2} d x+C \int(1+t)^{2 \lambda}\left(\left(p^{\prime}\left(v_{+}\right)-p^{\prime}(\bar{v})\right) \bar{v}_{x}\right)^{2} d x \\
& \quad \leq \frac{\alpha}{4} \int V_{t}^{2} d x+C \delta^{2} .
\end{aligned}
$$

Substituting (67)-(70) into (65), and noting the smallness of $\varepsilon$, we have

$$
\begin{aligned}
& \frac{1}{2} \frac{d}{d t} \int\left[(1+t)^{\lambda} V_{t}^{2}-(1+t)^{\lambda} p^{\prime}(\bar{v}) V_{x}^{2}\right] d x+\frac{\alpha}{4} \int V_{t}^{2} d x \\
& \leq \frac{d}{d t} \int(1+t)^{\lambda}\left(\int_{\bar{v}}^{V_{x}+\bar{v}+\hat{v}} p(s) d s-p(\bar{v}) V_{x}-\frac{1}{2} p^{\prime}(\bar{v}) V_{x}^{2}\right) d x \\
& \quad+C \int(1+t)^{\lambda-1}\left(V_{x}^{2}+V_{t}^{2}\right) d x+C \delta^{2} .
\end{aligned}
$$

Thus we have $(71) \times k+(64)$, and we have

$$
\begin{aligned}
& \frac{d}{d t} \int\left[V V_{t}+\frac{\alpha}{2}(1+t)^{-\lambda} V^{2}+\frac{k}{2}(1+t)^{\lambda} V_{t}^{2}-\frac{k}{2}(1+t)^{\lambda} p^{\prime}(\bar{v}) V_{x}^{2}\right] d x \\
& \quad+\frac{\alpha \lambda}{4} \int(1+t)^{-\lambda-1} V^{2} d x+\frac{C_{0}}{4} \int V_{x}^{2} d x+\frac{\alpha k-1}{4} \int V_{t}^{2} d x \\
& \leq k \\
& \quad k \frac{d}{d t} \int(1+t)^{\lambda}\left(\int_{\bar{v}}^{V_{x}+\bar{v}+\bar{v}} p(s) d s-p(\bar{v}) V_{x}-\frac{1}{2} p^{\prime}(\bar{v}) V_{x}^{2}\right) d x \\
& \quad+C k \int(1+t)^{\lambda-1}\left(V_{x}^{2}+V_{t}^{2}\right) d x+C \delta^{2} .
\end{aligned}
$$

Choosing $k=6 / \alpha$, using Gronwall's inequality on $[0, t]$ and the smallness of $\varepsilon$, we have

$\|V\|_{1}^{2}+\left\|V_{t}\right\|^{2}+\int_{0}^{t}\left(\left\|V_{x}\right\|^{2}+\left\|V_{t}\right\|^{2}\right) d s \leq C(T)\left(\left\|V_{0}\right\|_{1}^{2}+\left\|U_{0}\right\|^{2}+\delta\right)$.

This proves (53).

Step 2. Differentiating (57) $)_{1}$ with respect to $x$, one gets

$$
V_{x t t}+\left(p^{\prime}(\bar{v}) V_{x}\right)_{x x}+\frac{\alpha}{(1+t)^{\lambda}} V_{x t}=F_{x} .
$$

Multiplying (74) by $(1+t)^{\lambda} V_{x t}$ and integrating the resulting equation with respect to $x$ over $\mathbb{R}^{+}$, we can get

$$
\begin{aligned}
\frac{1}{2} \frac{d}{d t} \int & {\left[(1+t)^{\lambda} V_{x t}^{2}-(1+t)^{\lambda} p^{\prime}(\bar{v}) V_{x x}^{2}\right] d x+\alpha \int V_{x t}^{2} d x } \\
= & \frac{\lambda}{2} \int(1+t)^{\lambda-1} V_{x t}^{2} d x-\frac{\lambda}{2} \int(1+t)^{\lambda-1} p^{\prime}(\bar{v}) V_{x x}^{2} d x \\
& -\frac{1}{2} \int(1+t)^{\lambda} p^{\prime \prime}(\bar{v}) \bar{v}_{t} V_{x x}^{2} d x-\int(1+t)^{\lambda} p^{\prime \prime \prime}(\bar{v})\left|\bar{v}_{x}\right|^{2} V_{x} V_{x t} d x \\
& -\int(1+t)^{\lambda} p^{\prime \prime}(\bar{v}) \bar{v}_{x x} V_{x} V_{x t} d x-\int(1+t)^{\lambda} p^{\prime \prime}(\bar{v}) \bar{v}_{x} V_{x x} V_{x t} d x \\
& +\int(1+t)^{\lambda} F_{x} V_{x t} d x:=\sum_{k=1}^{7} I_{k} .
\end{aligned}
$$

By using Cauchy-Schwarz's inequality, Lemma 2.2, Corollary 2.3 and a priori assumption (51) to address the following estimates

$$
\begin{aligned}
& I_{1}+I_{2} \quad \leq C \int(1+t)^{\lambda-1}\left(V_{x t}^{2}+V_{x x}^{2}\right) d x, \\
& I_{3} \quad \leq C \delta \int(1+t)^{\lambda-1} V_{x x}^{2} d x, \\
& I_{4} \leq \frac{\alpha}{16} \int V_{x x}^{2} d x+C \int(1+t)^{2 \lambda}\left|\bar{v}_{x}\right|^{4} V_{x}^{2} d x \\
& \leq \frac{\alpha}{16} \int V_{x t}^{2} d x+C \varepsilon^{2} \delta^{2}, \\
& I_{5} \leq \frac{\alpha}{16} \int V_{x t}^{2} d x+C \int(1+t)^{2 \lambda}\left|\bar{v}_{x x}\right|^{2} V_{x}^{2} d x, \\
& \leq \frac{\alpha}{16} \int V_{x t}^{2} d x+C \varepsilon^{2} \delta^{2},
\end{aligned}
$$

and

$$
\begin{aligned}
I_{6} & \leq \frac{\alpha}{16} \int V_{x t}^{2} d x+C \int(1+t)^{2 \lambda}\left|\bar{v}_{x}\right|^{2} V_{x x}^{2} d x \\
& \leq \frac{\alpha}{16} \int V_{x t}^{2} d x+C \varepsilon^{2} \delta^{2} .
\end{aligned}
$$

Now, we turn to estimate $I_{7}$ as follows:

$$
\begin{aligned}
\int(1+t)^{\lambda} F_{x} V_{x t} t d x= & \int\left[\frac{(1+t)^{2 \lambda}}{\alpha} p^{\prime}\left(v_{+}\right) \bar{v}_{x x t}+\frac{\lambda(1+t)^{2 \lambda-1}}{\alpha} p^{\prime}\left(v_{+}\right) \bar{v}_{x x}\right. \\
& -(1+t)^{\lambda}\left(p\left(V_{x}+\bar{v}+\widehat{v}\right)-p(\bar{v})-p^{\prime}(\bar{v}) V_{x}\right)_{x x} \\
& \left.+(1+t)^{\lambda}\left(\left(p^{\prime}\left(v_{+}\right)-p^{\prime}(\bar{v})\right) \bar{v}_{x}\right)_{x}\right] V_{x t} d x:=\sum_{k=1}^{4} J_{k} .
\end{aligned}
$$

By using Lemma 2.1, Lemma 2.2, Corollary 2.3 and a priori assumption (51), we have

$J_{1} \leq \frac{\alpha}{16} \int V_{x t}^{2} d x+C \int(1+t)^{4 \lambda}\left|\bar{v}_{x x t}\right|^{2} d x \leq \frac{\alpha}{16} \int V_{x t}^{2} d x+C \delta^{2}$,

$J_{2} \leq \frac{\alpha}{16} \int V_{x t}^{2} d x+C \int(1+t)^{4 \lambda-2}\left|\bar{v}_{x x}\right|^{2} d x \leq \frac{\alpha}{16} \int V_{x t}^{2} d x+C \delta^{2}$, 


$$
\begin{aligned}
& J_{3}=\int(1+t)^{\lambda}\left(p\left(V_{x}+\bar{v}+\widehat{v}\right)-p(\bar{v})-p^{\prime}(\bar{v}) V_{x}\right)_{x} V_{x x t} d x \\
& \leq(1+t)^{\lambda} \frac{1}{2} \frac{d}{d t} \int\left(p^{\prime}\left(V_{x}+\bar{v}+\widehat{v}\right)-p^{\prime}(\bar{v})\right) V_{x x}^{2} d x \\
& -\frac{1}{2} \int(1+t)^{\lambda}\left(p^{\prime \prime}\left(V_{x}+\bar{v}+\widehat{v}\right)\left(V_{x t}+\bar{v}_{t}+\widehat{v}_{t}\right)-p^{\prime \prime}(\bar{v}) \bar{v}_{t}\right) V_{x x}^{2} d x \\
& +\int(1+t)^{\lambda}\left(p^{\prime}\left(V_{x}+\bar{v}+\widehat{v}\right)-p^{\prime}(\bar{v})-p^{\prime \prime}(\bar{v}) V_{x}\right) \bar{v}_{x} V_{x x t} d x \\
& +\int(1+t)^{\lambda} p^{\prime}\left(V_{x}+\bar{v}+\widehat{v}\right) \bar{v}_{x} V_{x x t} d x \\
& \leq \frac{1}{2} \frac{d}{d t} \int(1+t)^{\lambda}\left(p^{\prime}\left(V_{x}+\bar{v}+\widehat{v}\right)-p^{\prime}(\bar{v})\right) V_{x x}^{2} d x \\
& -\frac{\lambda}{2} \int(1+t)^{\lambda-1}\left(p^{\prime}\left(V_{x}+\bar{v}+\widehat{v}\right)-p^{\prime}(\bar{v})\right) V_{x x}^{2} d x \\
& +C(T)(\varepsilon+\delta) \int(1+t)^{\lambda-1} V_{x x}^{2} d x \\
& +\int(1+t)^{\lambda}\left(p^{\prime}\left(V_{x}+\bar{v}+\widehat{v}\right)-p^{\prime}(\bar{v})-p^{\prime \prime}(\bar{v}) V_{x}\right) \bar{v}_{x} V_{x x t} d x \\
& +\int(1+t)^{\lambda} p^{\prime}\left(V_{x}+\bar{v}+\widehat{v}\right) \widehat{v}_{x} V_{x x t} d x \\
& \leq \frac{1}{2} \frac{d}{d t} \int(1+t)^{\lambda}\left(p^{\prime}\left(V_{x}+\bar{v}+\widehat{v}\right)-p^{\prime}(\bar{v})\right) V_{x x}^{2} d x \\
& +\frac{\alpha}{16} \int V_{x t}^{2} d x+C(T)(\varepsilon+\delta) \int(1+t)^{\lambda-1} V_{x x}^{2} d x+C(T) \delta,
\end{aligned}
$$

and

$$
\begin{aligned}
J_{4} & \leq \frac{\alpha}{16} \int V_{x t}^{2} d x+C \int(1+t)^{2 \lambda}\left(\left(p^{\prime}\left(v_{+}\right)-p^{\prime}(\bar{v})\right) \bar{v}_{x}\right)_{x}^{2} d x \\
& \leq \frac{\alpha}{16} \int V_{x t}^{2} d x+C \delta^{2} .
\end{aligned}
$$

Substituting (76)-(85) into (75), and noticing $\varepsilon, \delta$ sufficiently small, we have

$$
\begin{aligned}
& \frac{1}{2} \frac{d}{d t} \int\left[(1+t)^{\lambda} V_{x t}^{2}-(1+t)^{\lambda} p^{\prime}(\bar{v}) V_{x x}^{2}\right] d x+\frac{\alpha}{2} \int V_{x t}^{2} d x \\
& \leq \frac{1}{2} \frac{d}{d t} \int(1+t)^{\lambda}\left(p^{\prime}\left(V_{x}+\bar{v}+\widehat{v}\right)-p^{\prime}(\bar{v})\right) V_{x x}^{2} d x \\
& \quad+C \int(1+t)^{\lambda-1}\left(V_{x x}^{2}+V_{x t}^{2}\right) d x+C(T) \delta .
\end{aligned}
$$

Multiplying (57) by $-V_{x x}$ and integrating the resulting equality with respect to $x$ over $\mathbb{R}^{+}$, we have

$$
\begin{aligned}
\frac{d}{d t} \int & {\left[V_{x} V_{x t}+\frac{\alpha}{2}(1+t)^{-\lambda} V_{x}^{2}\right] d x } \\
& +\frac{\alpha \lambda}{2} \int(1+t)^{-\lambda-1} V_{x}^{2} d x-\int p^{\prime}(\bar{v}) V_{x x}^{2} d x \\
= & \int V_{x t}^{2} d x+\int p^{\prime \prime}(\bar{v}) \bar{v}_{x} V_{x} V_{x x} d x-\int F V_{x x} d x
\end{aligned}
$$

It is easy to see that

$\int p^{\prime \prime}(\bar{v}) \bar{v}_{x} V_{x} V_{x x} d x \leq \frac{C_{0}}{12} \int V_{x x}^{2} d x+C \int\left|\bar{v}_{x}\right|^{2} V_{x}^{2} d x \leq \frac{C_{0}}{12} \int V_{x x}^{2} d x+C \varepsilon^{2} \delta^{2}$.
From (26), we have

$$
\begin{aligned}
-\int F V_{x x} d x= & -\int\left[\frac{(1+t)^{\lambda}}{\alpha} p^{\prime}\left(v^{+}\right) \bar{v}_{x t}+\frac{\lambda(1+t)^{\lambda-1}}{\alpha} p^{\prime}\left(v_{+}\right) \bar{v}_{x}\right. \\
& \left.-\left(p\left(V_{x}+\bar{v}+\widehat{v}\right)-p(\bar{v})-p^{\prime}(\bar{v}) V_{x}\right)_{x}+\left(p^{\prime}\left(v_{+}\right)-p^{\prime}(\bar{v})\right) \bar{v}_{x}\right] V_{x x} d x .
\end{aligned}
$$

By using Lemma 2.1, Lemma 2.2, Corollary 2.3 and a priori assumption (51), we have

$$
\begin{aligned}
& -\int \frac{(1+t)^{\lambda}}{\alpha} p^{\prime}\left(v_{+}\right) \bar{v}_{x t} V_{x x} d x \\
& \leq \frac{C_{0}}{12} \int V_{x x}^{2} d x+C \int(1+t)^{2 \lambda}\left|\bar{v}_{x t}\right|^{2} d x \\
& \quad \leq \frac{C_{0}}{12} \int V_{x x}^{2} d x+C \delta^{2}, \\
& -\int \frac{\lambda(1+t)^{\lambda-1}}{\alpha} p^{\prime}\left(v_{+}\right) \bar{v}_{x} V_{x x} d x \\
& \quad \leq \frac{C_{0}}{12} \int V_{x x}^{2} d x+C \int(1+t)^{2 \lambda-2}\left|\bar{v}_{x}\right|^{2} d x \\
& \leq \frac{C_{0}}{12} \int V_{x x}^{2} d x+C \delta^{2},
\end{aligned}
$$

$$
\begin{aligned}
& \int\left(p\left(V_{x}+\bar{v}+\widehat{v}\right)-p(\bar{v})-p^{\prime}(\bar{v}) V_{x}\right)_{x} V_{x x} d x \\
& =\int\left(p^{\prime}\left(V_{x}+\bar{v}+\widehat{v}\right)-p^{\prime}(\bar{v})\right) V_{x x}^{2} d x \\
& \quad+\int\left(p^{\prime}\left(V_{x}+\bar{v}+\widehat{v}\right)-p^{\prime}(\bar{v})-p^{\prime \prime}(\bar{v}) V_{x}\right) \bar{v}_{x} V_{x x} d x \\
& \quad+\int p^{\prime}\left(V_{x}+\bar{v}+\widehat{v}\right) \widehat{v}_{x} V_{x x} d x \\
& \leq C \int\left(\left|V_{x}\right|+|\widehat{v}|\right) V_{x x}^{2} d x+\frac{C_{0}}{12} \int V_{x x}^{2} d x \\
& \quad+\int\left|\bar{v}_{x}\right|^{2}\left(|\widehat{v}|^{2}+\left|V_{x}\right|^{4}\right) d x+C \int\left|\widehat{v}_{x}\right|^{2} d x \\
& \leq C(\varepsilon+\delta) \int V_{x x}^{2} d x+\frac{C_{0}}{12} \int V_{x x}^{2} d x+C \delta^{2},
\end{aligned}
$$

and

$$
\begin{aligned}
& \int\left(p^{\prime}\left(v_{+}\right)-p^{\prime}(\bar{v})\right) \bar{v}_{x} V_{x x} d x \\
& \leq \frac{C_{0}}{12} \int V_{x x}^{2} d x+C \int\left(\left(p^{\prime}\left(v_{+}\right)-p^{\prime}(\bar{v})\right) \bar{v}_{x}\right)^{2} d x \\
& \leq \frac{C_{0}}{12} \int V_{x x}^{2} d x+C \delta^{2} .
\end{aligned}
$$

Substituting (88)-(93) into (87), and using the smallness of $\delta$, $\varepsilon$, we have

$$
\begin{aligned}
& \frac{d}{d t} \int\left[V_{x} V_{x t}+\frac{\alpha}{2}(1+t)^{-\lambda} V_{x}^{2}\right] d x \\
& \quad+\frac{\alpha \lambda}{2} \int(1+t)^{-\lambda-1} V_{x}^{2} d x+\frac{C_{0}}{2} \int V_{x x}^{2} d x \\
& \leq \int V_{x t}^{2} d x+C \delta^{2} .
\end{aligned}
$$


Multiplying (86) by $h$ and adding up the resulting inequality and (94), we get

$$
\begin{aligned}
& \frac{d}{d t} \int\left[V_{x} V_{x t}+\frac{\alpha}{2}(1+t)^{-\lambda} V_{x}^{2}+\frac{h}{2}(1+t)^{\lambda} V_{x t}^{2}-\frac{h}{2}(1+t)^{\lambda} p^{\prime}(\bar{v}) V_{x x}^{2}\right] d x \\
& \quad+\frac{\alpha \lambda}{2} \int(1+t)^{-\lambda-1} V_{x}^{2} d x+\frac{C_{0}}{2} \int V_{x x}^{2} d x+\left(\frac{\alpha h}{2}-1\right) \int V_{x t}^{2} d x \\
& \leq \frac{h}{2} \frac{d}{d t} \int(1+t)^{\lambda}\left(p^{\prime}\left(V_{x}+\bar{v}+\widehat{v}\right)-p^{\prime}(\bar{v})\right) V_{x x}^{2} d x \\
& \quad+C h \int(1+t)^{\lambda-1}\left(V_{x x}^{2}+V_{x t}^{2}\right) d x+C \delta^{2} .
\end{aligned}
$$

Taking $h=6 / \alpha$, using Gronwall's inequality on $[0, t]$ and (53), we have

$\left\|V_{x x}\right\|^{2}+\left\|V_{x t}\right\|^{2}+\int_{0}^{t}\left(\left\|V_{x x}\right\|^{2}+\left\|V_{x t}\right\|^{2}\right) d s \leq C(T)\left(\left\|V_{0}\right\|_{2}^{2}+\left\|U_{0}\right\|_{1}^{2}+\delta\right)$.

This proves (154).

Step 3. Similar calculations to (73) and (96), we can get the high order estimate (3.5). The details are omitted.

From (53), (54), and (55), by using the continuity technique, we can verify the a priori assumption (51) is true provided $\left\|V_{0}\right\|_{3}^{2}+\left\|U_{0}\right\|_{2}^{2}+\delta$ sufficiently small. The proof of Proposition 3.1 is complete.

\section{Decay Rates}

The main goal of this section is to obtain the decay rates of the solution $(V, U)(x, t)$. We devote ourselves to the estimates of the solution $(V, U)(x, t)$ under the a priori assumption

$$
\begin{aligned}
N_{1}(T):= & \sup _{0<t<T}\left\{\left\|V_{x}(\cdot, t)\right\|_{L^{\infty}}+(1+t)\left\|V_{x t}(\cdot, t)\right\|_{L^{\infty}}\right. \\
& \left.+(1+t)^{\lambda+1 / 2}\left\|V_{x x}(\cdot, t)\right\|_{L^{\infty}}\right\} \leq \varepsilon,
\end{aligned}
$$

where $0<\varepsilon \ll 1,0<T<\infty$.

Lemma 4.1. Under the assumptions of Theorem 2.4, if $\varepsilon$ is small enough, it holds that

$$
\begin{gathered}
\|V\|^{2}+(1+t)^{2 \lambda}\left(\left\|V_{t}\right\|^{2}+\left\|V_{x}\right\|^{2}\right)+\int_{0}^{t}(1+s)^{\lambda}\left(\left\|V_{t}\right\|^{2}+\left\|V_{x}\right\|^{2}\right) d s \\
\leq C\left(\left\|V_{0}\right\|_{1}^{2}+\left\|U_{0}\right\|^{2}+\delta\right), \quad \text { for } 0 \leq \lambda<\frac{3}{5} .
\end{gathered}
$$

For $3 / 5<\lambda<1$, we have

$$
\begin{aligned}
& (1+t)^{(3 / 2)-(5 \lambda / 2)}\|V\|^{2}+(1+t)^{(3 / 2)-(\lambda / 2)}\left(\left\|V_{t}\right\|^{2}+\left\|V_{x}\right\|^{2}\right) \\
& \quad \leq C\left(\left\|V_{0}\right\|_{1}^{2}+\left\|U_{0}\right\|^{2}+\delta\right),
\end{aligned}
$$

and

$$
\begin{gathered}
\int_{0}^{t}\left[(1+s)^{\beta-\lambda-1}\|V\|^{2}+(1+s)^{\beta}\left(\left\|V_{t}\right\|^{2}+\left\|V_{x}\right\|^{2}\right)\right] d s \\
\leq C(1+t)^{\beta+(3 \lambda / 2)-(3 / 2)}\left(\left\|V_{0}\right\|_{1}^{2}+\left\|U_{0}\right\|^{2}+\delta\right),
\end{gathered}
$$

for any $3 / 2-3 \lambda / 2<\beta<\lambda$.

Proof. Multiplying (57) $)_{1}$ by $(1+t)^{\beta} V$ and integrating the resulting equality with respect to $x$ over $\mathbb{R}^{+}$, we obtain

$$
\begin{aligned}
& \frac{d}{d t} \int\left[(1+t)^{\beta} V V_{t}+\frac{\alpha}{2}(1+t)^{\beta-\lambda} V^{2}\right] d x \\
& \quad+\frac{\alpha(\lambda-\beta)}{2} \int(1+t)^{\beta-\lambda-1} V^{2} d x-\int(1+t)^{\beta} p^{\prime}(\bar{v}) V_{x}^{2} d x \\
& =\int(1+t)^{\beta} V_{t}^{2} d x+\frac{\beta}{2} \frac{d}{d t} \int(1+t)^{\beta-1} V^{2} d x \\
& \quad+\frac{\beta(1-\beta)}{2} \int(1+t)^{\beta-2} V^{2} d x+\int(1+t)^{\beta} F V d x
\end{aligned}
$$

Now, we estimate the last term in the right hand of (101), from (39), we have

$$
\begin{aligned}
& \int(1+t)^{\beta} F V d x=\int\left[\frac{(1+t)^{\beta+\lambda}}{\alpha} p^{\prime}\left(v_{+}\right) \bar{v}_{x t}+\frac{\lambda(1+t)^{\beta+\lambda-1}}{\alpha}\right. \\
& \cdot p^{\prime}\left(v_{+}\right) \bar{v}_{x}-(1+t)^{\beta}\left(p\left(V_{x}+\bar{v}+\widehat{v}\right)-p(\bar{v})-p^{\prime}(\bar{v}) V_{x}\right) \\
& \left.+(1+t)^{\beta}\left(p^{\prime}(\bar{v}+)-p^{\prime}(\bar{v})\right) \bar{v}_{x}\right] V d x .
\end{aligned}
$$

From Lemma 2.2, the Cauchy-Schwarz's inequality and noticing $p^{\prime}(v) \leq-C_{0}<0$, we have

$$
\begin{aligned}
\int \frac{(1+t)^{\beta+\lambda}}{\alpha} p^{\prime}\left(v_{+}\right) \bar{v}_{x t} V d x & =-\int \frac{(1+t)^{\beta+\lambda}}{\alpha} p^{\prime}\left(v_{+}\right) \bar{v}_{t} V_{x} d x \\
& \leq \frac{C_{0}}{8} \int(1+t)^{\beta} V_{x}^{2} d x+C \int(1+t)^{\beta+2 \lambda}\left|\bar{v}_{t}\right|^{2} d x \\
& \leq \frac{C_{0}}{8} \int(1+t)^{\beta} V_{x}^{2} d x+C \delta^{2}(1+t)^{\beta+(3 \lambda) / 2)-(5 / 2)},
\end{aligned}
$$

and for some constant $\kappa>1, v>0$, which will be determined below

$$
\begin{aligned}
& \int \frac{\lambda(1+t)^{\beta+\lambda-1}}{\alpha} p^{\prime}\left(v_{+}\right) \bar{v}_{x} V d x \\
& \leq v \int(1+t)^{-\kappa} V^{2} d x+\frac{C}{v} \int(1+t)^{2 \beta+2 \lambda+\kappa-2}\left|\bar{v}_{x}\right|^{2} d x \\
& \leq v \int(1+t)^{-\kappa} V^{2} d x+\frac{C \delta^{2}}{v}(1+t)^{2 \beta+\kappa+(\lambda / 2)-(7 / 2)} .
\end{aligned}
$$

By using Lemmas 2.1, Lemma 2.2 and a priori assumption (97), we have

$$
\begin{aligned}
& -\int(1+t)^{\beta}\left(p\left(V_{x}+\bar{v}+\widehat{v}\right)-p(\bar{v})-p^{\prime}(\bar{v}) V_{x}\right)_{x} V d x \\
& =\int(1+t)^{\beta}\left(p\left(V_{x}+\bar{v}+\widehat{v}\right)-p(\bar{v})-p^{\prime}(\bar{v}) V_{x}\right) V_{x} d x \\
& \quad \leq \frac{C_{0}}{8} \int(1+t)^{\beta} V_{x}^{2} d x+C \int(1+t)^{\beta}\left(|\widehat{v}|^{2}+\left|V_{x}\right|^{4}+|\widehat{v}|^{4}\right) d x \\
& \quad \leq \frac{C_{0}}{8} \int(1+t)^{\beta} V_{x}^{2} d x+C \varepsilon^{2} \int(1+t)^{\beta} V_{x}^{2} d x+C \delta^{2} e^{-c t 9},
\end{aligned}
$$


and

$$
\begin{aligned}
& \int(1+t)^{\beta}\left(p^{\prime}\left(v_{+}\right)-p^{\prime}(\bar{v})\right) \bar{v}_{x} V d x \\
& \leq v \int(1+t)^{-\kappa} V^{2} d x \\
& \quad+\frac{C}{v} \int\left(\left(p^{\prime}\left(v_{+}\right)-p^{\prime}(\bar{v})\right) \bar{v}_{x}\right)^{2}(1+t)^{2 \beta+\kappa} d x \\
& \leq v \int(1+t)^{-\kappa} V^{2} d x+\frac{C \delta^{2}}{v}(1+t)^{2 \beta+\kappa-(5 \lambda / 2)-(5 / 2)} .
\end{aligned}
$$

Substituting (102)-(106) into (101), and noting the smallness of $\varepsilon$, we have

$$
\begin{aligned}
& \frac{d}{d t} \int\left[(1+t)^{\beta} V V_{t}+\frac{\alpha}{2}(1+t)^{\beta-\lambda} V^{2}\right] d x+\frac{\alpha(\lambda-\beta)}{2} \int(1+t)^{\beta-\lambda-1} V^{2} d x \\
& +\frac{C_{0}}{2} \int(1+t)^{\beta} V_{x}^{2} d x \leq \int(1+t)^{\beta} V_{t}^{2} d x+\frac{\beta}{2} \frac{d}{d t} \int(1+t)^{\beta-1} V^{2} d x \\
& +\frac{\beta(1-\beta)}{2} \int(1+t)^{\beta-2} V^{2} d x+2 v \int(1+t)^{-\kappa} V^{2} d x \\
& +C \delta^{2}(1+t)^{\beta+(3 \lambda / 2)-(5 / 2)}+\frac{C \delta^{2}}{v}(1+t)^{2 \beta+\kappa+(\lambda / 2)-(7 / 2)} \\
& \quad+\frac{C \delta^{2}}{v}(1+t)^{2 \beta+\kappa-(5 / / 2)-(7 / 2)} .
\end{aligned}
$$

Next, multiplying (57) ${ }_{1}$ by $(1+t)^{\beta+\lambda} V_{t}$ and integrating the resulting equation with respect to $x$ over $\mathbb{R}^{+}$, we can get

$$
\begin{aligned}
& \frac{1}{2} \frac{d}{d t} \int\left[(1+t)^{\beta+\lambda} V_{t}^{2}-(1+t)^{\beta+\lambda} p^{\prime}(\bar{v}) V_{x}^{2}\right] d x \\
& \quad+\int \alpha(1+t)^{\beta} V_{t}^{2} d x=-\frac{1}{2} \int(1+t)^{\beta+\lambda} p^{\prime \prime}(\bar{v}) \bar{v}_{t} V_{x}^{2} d x \\
& \quad-\frac{\beta+\lambda}{2} \int(1+t)^{\beta+\lambda-1} p^{\prime}(\bar{v}) V_{x}^{2} d x+\frac{\beta+\lambda}{2} \int(1+t)^{\beta+\lambda-1} V_{t}^{2} d x \\
& \quad+\int(1+t)^{\beta+\lambda} F V_{t} d x \leq C \int(1+t)^{\beta+\lambda-1}\left(V_{x}^{2}+V_{t}^{2}\right) d x \\
& \quad+\int(1+t)^{\beta+\lambda} F V_{t} d x .
\end{aligned}
$$

Now, we estimate the last term in the right hand of (108) as follows:

$$
\begin{aligned}
\int(1+t)^{\beta+\lambda} F V_{t} d x & =\int\left[\frac{(1+t)^{\beta+2 \lambda}}{\alpha} p^{\prime}\left(v_{+}\right) \bar{v}_{x t}+\frac{\lambda(1+t)^{\beta+2 \lambda-1}}{\alpha} p^{\prime}\left(v_{+}\right) \bar{v}_{x}\right. \\
& -(1+t)^{\beta+\lambda}\left(p\left(V_{x}+\bar{v}+\widehat{v}\right)-p(\bar{v})-p^{\prime}(\bar{v}) V_{x}\right)_{x} \\
& \left.+(1+t)^{\beta+\lambda}\left(p^{\prime}\left(v_{+}\right)-p^{\prime}(\bar{v})\right) \bar{v}_{x}\right] V_{t} d x .
\end{aligned}
$$

By using Lemma 2.1, Lemma 2.2 and a priori assumption (97), we have

$$
\begin{aligned}
& \int \frac{(1+t)^{\beta+2 \lambda}}{\alpha} p^{\prime}\left(v_{+}\right) \bar{v}_{x t} V_{t} d x \\
& \leq \frac{\alpha}{4} \int(1+t)^{\beta} V_{t}^{2} d x+C \int(1+t)^{\beta+4 \lambda}\left|\bar{v}_{x t}\right|^{2} d x \\
& \leq \frac{\alpha}{4} \int(1+t)^{\beta} V_{t}^{2} d x+C \delta^{2}(1+t)^{\beta+(5 \lambda / 2)-(7 / 2)}, \\
& \int \frac{\lambda(1+t)^{\beta+2 \lambda-1}}{\alpha} p^{\prime}\left(v_{+}\right) \bar{v}_{x} V_{t} d x \\
& \quad \leq \frac{\alpha}{4} \int(1+t)^{\beta} V_{t}^{2} d x+C \int(1+t)^{\beta+4 \lambda-2}\left|\bar{v}_{x}\right|^{2} d x \\
& \quad \leq \frac{\alpha}{4} \int(1+t)^{\beta} V_{t}^{2} d x+C \delta^{2}(1+t)^{\beta+(5 \lambda / 2)-(7 / 2)},
\end{aligned}
$$

$$
\begin{aligned}
- & \int(1+t)^{\beta+\lambda}\left(p\left(V_{x}+\bar{v}+\widehat{v}\right)-p(\bar{v})-p^{\prime}(\bar{v}) V_{x}\right)_{x} V_{t} d x \\
= & \int(1+t)^{\beta+\lambda}\left(p\left(V_{x}+\bar{v}+\widehat{v}\right)-p(\bar{v})-p^{\prime}(\bar{v}) V_{x}\right) V_{x t} d x \\
= & (1+t)^{\beta+\lambda} \frac{d}{d t} \int\left(\int_{\bar{v}}^{V_{x}+\bar{v}+\hat{v}} p(s) d s-p(\bar{v}) V_{x}-\frac{1}{2} p^{\prime}(\bar{v}) V_{x}^{2}\right) d x \\
& -\int(1+t)^{\beta+\lambda}\left(p\left(V_{x}+\bar{v}+\widehat{v}\right)-p(\bar{v})-p^{\prime}(\bar{v}) V_{x}-\frac{1}{2} p^{\prime \prime}(\bar{v}) V_{x}^{2}\right) \bar{v}_{t} d x \\
& -\int(1+t)^{\beta+\lambda} p\left(V_{x}+\bar{v}+\hat{v}\right) \hat{v}_{t} d x \\
\leq & \frac{d}{d t} \int(1+t)^{\beta+\lambda}\left(\int_{\bar{v}}^{V_{x}+\bar{v}+\hat{v}} p(s) d s-p(\bar{v}) V_{x}-\frac{1}{2} p^{\prime}(\bar{v}) V_{x}^{2}\right) d x \\
& -(\beta+\lambda) \int(1+t)^{\beta+\lambda-1}\left(\int_{\bar{v}}^{V_{x}+\bar{v}+\hat{v}} p(s) d s-p(\bar{v}) V_{x}-\frac{1}{2} p^{\prime}(\bar{v}) V_{x}^{2}\right) d x \\
& +C \delta \int(1+t)^{\beta+\lambda-1}\left(|\hat{v}|+\left|V_{x}\right|^{3}\right) d x+C \int(1+t)^{\beta+\lambda}\left|\hat{v}_{t}\right| d x \\
\leq & \frac{d}{d t} \int(1+t)^{\beta+\lambda}\left(\int_{\bar{v}}^{V_{x}+\bar{v}+\bar{v}} p(s) d s-p(\bar{v}) V_{x}-\frac{1}{2} p^{\prime}(\bar{v}) V_{x}^{2}\right) d x \\
& +C(\varepsilon+\delta) \int(1+t)^{\beta+\lambda-1} V_{x}^{2} d x+C \delta^{2} e^{-c t^{\theta}}(1+t)^{\beta+\lambda},
\end{aligned}
$$

and

$\int(1+t)^{\beta+\lambda}\left(p^{\prime}\left(v_{+}\right)-p^{\prime}(\bar{v})\right) \bar{v}_{x} V_{t} d x$

$\leq \frac{\alpha}{4} \int(1+t)^{\beta} V_{t}^{2} d x+C \int(1+t)^{\beta+2 \lambda}\left(\left(p^{\prime}\left(v_{+}\right)-p^{\prime}(\bar{v})\right) \bar{v}_{x}\right)^{2} d x$

$\leq \frac{\alpha}{4} \int(1+t)^{\beta} V_{t}^{2} d x+C \delta^{2}(1+t)^{\beta-(\lambda / 2)-(5 / 2)}$.

Substituting (110)-(113) into (108), and noting the smallness of $\varepsilon$, we have

$$
\begin{aligned}
& \frac{1}{2} \frac{d}{d t} \int\left[(1+t)^{\beta+\lambda} V_{t}^{2}-(1+t)^{\beta+\lambda} p^{\prime}(\bar{v}) V_{x}^{2}\right] d x+\frac{\alpha}{4} \int(1+t)^{\beta} V_{t}^{2} d x \\
& \leq \frac{d}{d t} \int(1+t)^{\beta+\lambda}\left(\int_{\bar{v}}^{V_{x}+\bar{v}+\hat{v}} p(s) d s-p(\bar{v}) V_{x}-\frac{1}{2} p^{\prime}(\bar{v}) V_{x}^{2}\right) d x \\
& \quad+C \int(1+t)^{\beta+\lambda-1}\left(V_{x}^{2}+V_{t}^{2}\right) d x+C \delta^{2}(1+t)^{\beta+(5 \lambda / 2)-(7 / 2)} \\
& \quad+C \delta^{2}(1+t)^{\beta-(\lambda / 2)-(5 / 2) .}
\end{aligned}
$$

Thus we have $(114) \times h+(107)$, and we have

$$
\begin{aligned}
& \frac{d}{d t} \int\left[(1+t)^{\beta} V V_{t}+\frac{\alpha}{2}(1+t)^{\beta-\lambda} V^{2}\right. \\
& \left.+\frac{h}{2}(1+t)^{\beta+\lambda} V_{t}^{2}-\frac{h}{2}(1+t)^{\beta+\lambda} p^{\prime}(\bar{v}) V_{x}^{2}\right] d x \\
& +\frac{\alpha(\lambda-\beta)}{2} \int(1+t)^{\beta-\lambda-1} V^{2} d x+\frac{C_{0}}{2} \int(1+t)^{\beta} V_{x}^{2} d x \\
& +\left(\frac{\alpha h}{4}-1\right) \int(1+t)^{\beta} V_{t}^{2} d x \\
& \leq \\
& \quad h \frac{d}{d t} \int(1+t)^{\beta+\lambda}\left(\int_{\bar{v}}^{V_{x}+\bar{v}+\hat{v}} p(s) d s-p(\bar{v}) V_{x}-\frac{1}{2} p^{\prime}(\bar{v}) V_{x}^{2}\right) d x \\
& +\frac{\beta}{2} \frac{d}{d t} \int(1+t)^{\beta-1} V^{2} d x+\frac{\beta(1-\beta)}{2} \int(1+t)^{\beta-2} V^{2} d x \\
& +2 v \int(1+t)^{-\kappa} V^{2} d x+C h \int(1+t)^{\beta+\lambda-1}\left(V_{x}^{2}+V_{t}^{2}\right) d x \\
& +C \delta^{2}(1+t)^{\beta+(3 \lambda / 2)-(5 / 2)}+\frac{C \delta^{2}}{v}(1+t)^{2 \beta+\kappa+(\lambda / 2)-(7 / 2)} \\
& +\frac{C \delta^{2}}{v}(1+t)^{2 \beta+\kappa-(5 \lambda / 2)-(5 / 2) .}
\end{aligned}
$$


Case $1(0 \leq \lambda<3 / 5)$. It is easy to know that $1<5 / 2-5 \lambda / 2$. Therefore, we can take $\beta=\lambda, v=1 / 2$, and there exist constant $\kappa$ satisfying $1<\kappa<\min \{5 / 2-5 \lambda / 2, \lambda / 2+3 / 2,2-\lambda\}$. Then, we have

$$
\begin{aligned}
& \frac{d}{d t} \int\left[(1+t)^{\lambda} V V_{t}+\frac{\alpha}{2} V^{2}+\frac{h}{2}(1+t)^{2 \lambda} V_{t}^{2}-\frac{h}{2}(1+t)^{2 \lambda} p^{\prime}(\bar{v}) V_{x}^{2}\right] d x \\
& \quad+\frac{C_{0}}{2} \int(1+t)^{\lambda} V_{x}^{2} d x+\left(\frac{\alpha h}{4}-1\right) \int(1+t)^{\lambda} V_{t}^{2} d x \\
& \leq h \frac{d}{d t} \int(1+t)^{2 \lambda}\left(\int_{\bar{v}}^{V_{x}+\bar{v}+\hat{v}} p(s) d s-p(\bar{v}) V_{x}-\frac{1}{2} p^{\prime}(\bar{v}) V_{x}^{2}\right) d x \\
& \quad+\frac{\lambda}{2} \frac{d}{d t} \int(1+t)^{\lambda-1} V^{2} d x+\frac{\lambda(1-\lambda)}{2} \int(1+t)^{\lambda-2} V^{2} d x \\
& \quad+\int(1+t)^{-\kappa} V^{2} d x+C h \int(1+t)^{2 \lambda-1}\left(V_{x}^{2}+V_{t}^{2}\right) d x \\
& \quad+C \delta^{2}(1+t)^{\kappa+(5 \lambda / 2)-(7 / 2)}+C \delta^{2}(1+t)^{\kappa-(\lambda / 2)-(5 / 2) .}
\end{aligned}
$$

Let $T_{0}$ be sufficiently large such that if $t \geq T_{0}$, it holds

$$
\left\{\begin{array}{l}
C h(1+t)^{\lambda-1} \leq \frac{C_{0}}{4}, \\
C h(1+t)^{\lambda-1} \leq \frac{1}{2}\left(\frac{\alpha h}{4}-1\right), \\
\frac{\lambda}{2}(1+t)^{\lambda-1} \leq \frac{1}{4} .
\end{array}\right.
$$

Fixing $h=12 / \alpha$, from (116) and (117) we have $\frac{d}{d t} H(t)+\frac{C_{0}}{2} \int(1+t)^{\lambda} V_{x}^{2} d x+\left(\frac{\alpha h}{4}-1\right) \int(1+t)^{\lambda} V_{t}^{2} d x$ $\leq C \frac{d}{d t} \int(1+t)^{2 \lambda}\left(\int_{\bar{v}}^{V_{x}+\bar{v}+\hat{v}} p(s) d s-p(\bar{v}) V_{x}-\frac{1}{2} p^{\prime}(\bar{v}) V_{x}^{2}\right) d x$

$+\frac{\lambda}{2} \frac{d}{d t} \int(1+t)^{\lambda-1} V^{2} d x+C \int(1+t)^{-\kappa} V^{2} d x+\frac{C_{0}}{4} \int(1+t)^{\lambda} V_{x}^{2} d x$ $+\frac{1}{2}\left(\frac{\alpha h}{4}-1\right) \int(1+t)^{\lambda} V_{t}^{2} d x+C \delta^{2}(1+t)^{\kappa+(5 \lambda / 2)-(7 / 2)}$

$+C \delta^{2}(1+t)^{\kappa-(\lambda / 2)-(5 / 2)}$,

where

$$
H(t) \sim\|V\|^{2}+(1+t)^{2 \lambda}\left\|V_{x}\right\|^{2}+(1+t)^{2 \lambda}\left\|V_{t}\right\|^{2} .
$$

For any $t \in\left[T_{0},+\infty\right)$, we have

$$
\begin{aligned}
& \frac{d}{d t} H(t)+\frac{C_{0}}{4} \int(1+t)^{\lambda} V_{x}^{2} d x+\frac{1}{2}\left(\frac{\alpha h}{4}-1\right) \int(1+t)^{\lambda} V_{t}^{2} d x \\
& \leq C \frac{d}{d t} \int(1+t)^{2 \lambda}\left(\int_{\bar{v}}^{V_{x}+\bar{v}+\widehat{v}} p(s) d s-p(\bar{v}) V_{x}-\frac{1}{2} p^{\prime}(\bar{v}) V_{x}^{2}\right) d x \\
& \quad+\frac{\lambda}{2} \frac{d}{d t} \int(1+t)^{\lambda-1} V^{2} d x+C(1+t)^{-\kappa} H(t) \\
& \quad+C \delta^{2}(1+t)^{\kappa+(5 \lambda / 2)-(7 / 2)}+C \delta^{2}(1+t)^{\kappa-(\lambda / 2)-(5 / 2)} .
\end{aligned}
$$

Using (117) and Gronwall's inequality on $\left[T_{0}, t\right]$, noting $1<\kappa<\min \{5 / 2-5 \lambda / 2, \lambda / 2+3 / 2\}, 0 \leq \lambda<(3 / 5)$, we have

$$
\begin{aligned}
H(t)+ & C \int_{T_{0}}^{t}(1+s)^{\lambda}\left(\left\|V_{x}\right\|^{2}+\left\|V_{t}\right\|^{2}\right) d s \\
\leq & C \int(1+t)^{2 \lambda}\left(\int_{\bar{v}}^{V_{x}+\bar{v}+\hat{v}} p(s) d s-p(\bar{v}) V_{x}-\frac{1}{2} p^{\prime}(\bar{v}) V_{x}^{2}\right) d x \\
& +\frac{\lambda}{2} \int(1+t)^{\lambda-1} V^{2} d x+C\left(H\left(T_{0}\right)+\delta\right) \\
\leq & C \varepsilon \int(1+t)^{2 \lambda}\left|V_{x}\right|^{2} d x+\frac{1}{4} \int V^{2} d x+C\left(H\left(T_{0}\right)+\delta\right),
\end{aligned}
$$

which together with (73) and Proposition 3.1 deduce (98) in view of the smallness of $\varepsilon$.

Case $2(3 / 5<\lambda<1)$. In this case, it is easy to know that $\lambda>(3 / 2)-(3 \lambda / 2)$. Therefore, we can take $3 / 2-3 \lambda / 2<\beta<\lambda, \kappa=\lambda-\beta+1$, and $\nu=\alpha(\lambda-\beta) / 8>0$. Then, from (115), we have

$$
\begin{aligned}
& \frac{d}{d t} \int\left[(1+t)^{\beta} V V_{t}+\frac{\alpha}{2}(1+t)^{\beta-\lambda} V^{2}\right. \\
& \left.\quad+\frac{h}{2}(1+t)^{\beta+\lambda} V_{t}^{2}-\frac{h}{2}(1+t)^{\beta+\lambda} p^{\prime}(\bar{v}) V_{x}^{2}\right] d x \\
& \quad+\frac{\alpha(\lambda-\beta)}{4} \int(1+t)^{\beta-\lambda-1} V^{2} d x+\frac{C_{0}}{2} \int(1+t)^{\beta} V_{x}^{2} d x \\
& \quad+\left(\frac{\alpha h}{4}-1\right) \int(1+t)^{\beta} V_{t}^{2} d x \\
& \leq \\
& +\frac{d}{d t} \int(1+t)^{\beta+\lambda}\left(\int_{\bar{v}}^{V_{x}+\bar{v}+\hat{v}} p(s) d s-p(\bar{v}) V_{x}-\frac{1}{2} p^{\prime}(\bar{v}) V_{x}^{2}\right) d x \\
& \quad+\frac{\beta}{2} \frac{d}{d t} \int(1+t)^{\beta-1} V^{2} d x+\frac{\beta(1-\beta)}{2} \int(1+t)^{\beta-2} V^{2} d x \\
& \quad+C h \int(1+t)^{\beta+\lambda-1}\left(V_{x}^{2}+V_{t}^{2}\right) d x+C \delta^{2}(1+t)^{\beta+(3 \lambda / 2)-(5 / 2)} .
\end{aligned}
$$

Let $T_{1}$ be sufficiently large such that if $t \geq T_{1}$, it holds

$$
\left\{\begin{array}{l}
\operatorname{Ch}(1+t)^{\lambda-1} \leq \frac{C_{0}}{4}, \\
\operatorname{Ch}(1+t)^{\lambda-1} \leq \frac{1}{2}\left(\frac{\alpha h}{4}-1\right), \\
\frac{\beta}{2}(1+t)^{\lambda-1} \leq \frac{1}{4} .
\end{array}\right.
$$

Fixing $h=12 / \alpha$, from (112), we have

$$
\begin{aligned}
& \frac{d}{d t} H_{1}(t)+\frac{C_{0}}{4} \int(1+t)^{\beta} V_{x}^{2} d x+\frac{1}{2}\left(\frac{\alpha h}{4}-1\right) \int(1+t)^{\beta} V_{t}^{2} d x \\
& +\frac{\alpha(\lambda-\beta)}{4} \int(1+t)^{\beta-\lambda-1} V^{2} d x \leq C \frac{d}{d t} \int(1+t)^{\beta+\lambda} \\
& \quad \cdot\left(\int_{\bar{v}}^{V_{x}+\bar{v}+\bar{v}} p(s) d s-p(\bar{v}) V_{x}-\frac{1}{2} p^{\prime}(\bar{v}) V_{x}^{2}\right) d x+\frac{\beta}{2} \frac{d}{d t} \int(1+t)^{\beta-1} V^{2} d x \\
& +\frac{\beta(1-\beta)}{2} \int(1+t)^{\beta-2} V^{2} d x+C \delta^{2}(1+t)^{\beta+(3 \lambda / 2)-(5 / 2),}
\end{aligned}
$$

where

$$
H_{1}(t) \sim(1+t)^{\beta-\lambda}\|V\|^{2}+(1+t)^{\beta+\lambda}\left\|V_{x}\right\|^{2}+(1+t)^{\beta+\lambda}\left\|V_{t}\right\|^{2} .
$$

Then, from (124), we know

$$
\begin{aligned}
\frac{d}{d t} H_{1}(t)+ & \frac{C_{0}}{4} \int(1+t)^{\beta} V_{x}^{2} d x+C \int(1+t)^{\beta} V_{t}^{2} d x \\
+ & C \int(1+t)^{\beta-\lambda-1} V^{2} d x \leq C \frac{d}{d t} \int(1+t)^{\beta+\lambda} \\
& \cdot\left(\int_{\bar{v}}^{V_{x}+\bar{v}+\bar{v}} p(s) d s-p(\bar{v}) V_{x}-\frac{1}{2} p^{\prime}(\bar{v}) V_{x}^{2}\right) d x \\
+ & \frac{\beta}{2} \frac{d}{d t} \int(1+t)^{\beta-1} V^{2} d x \\
+ & C(1+t)^{\lambda-2} H_{1}(t)+C \delta^{2}(1+t)^{\beta+(3 \lambda / 2)-(5 / 2)}
\end{aligned}
$$


for any $t \in\left[T_{1}, \infty\right)$.

Using Gronwall's inequality on $\left[T_{1}, t\right],(123)$ and noting $\beta+3 \lambda / 2-5 / 2>-1,3 / 5<\lambda<1$, we have

$$
\begin{aligned}
H_{1}(t)+ & C \int_{T_{1}}^{t}\left[(1+s)^{\beta}\left(\left\|V_{x}\right\|^{2}+\left\|V_{t}\right\|^{2}\right)+(1+s)^{\beta-\lambda-1}\|V\|^{2}\right] d s \\
\leq & C \int(1+t)^{\beta+\lambda}\left\{\int_{\bar{v}}^{V_{x}+\bar{v}+\hat{v}} p(s) d s-p(\bar{v}) V_{x}-\frac{1}{2} p^{\prime}(\bar{v}) V_{x}^{2}\right\} d x \\
& +\frac{\beta}{2} \int(1+t)^{\beta-1} V^{2} d x+H_{1}\left(T_{1}\right)+C \delta^{2}(1+t)^{\beta+(3 \lambda / 2)-(3 / 2)} \\
\leq & C \varepsilon \int(1+t)^{\beta+\lambda}\left|V_{x}\right|^{2} d x+\frac{1}{4} \int(1+t)^{\beta-\lambda} V^{2} d x \\
& +H_{1}\left(T_{1}\right)+C \delta^{2}(1+t)^{\beta+(3 \lambda / 2)-(3 / 2)},
\end{aligned}
$$

which together with (73) and Proposition 3.1 deduce (99) and (100), in view of the smallness of $\varepsilon$ and $\beta+3 \lambda / 2-3 / 2>0$.

Hence, we complete the proof of Lemma 4.1.

Furthermore, we can get the better decay rate of the functions $V_{x}$ and $V_{t}$ as follows.

Lemma 4.2. Under the assumptions of Theorem 2.4, if $\varepsilon$ is small enough, it holds that

$$
\begin{aligned}
& (1+t)^{\lambda+1}\left(\left\|V_{t}\right\|^{2}+\left\|V_{x}\right\|^{2}\right)+\int_{0}^{t}(1+s)\left\|V_{t}\right\|^{2} d s \\
& \leq C\left(\left\|V_{0}\right\|_{1}^{2}+\left\|U_{0}\right\|^{2}+\delta\right), \quad \text { for } \quad 0 \leq \lambda<\frac{3}{5} .
\end{aligned}
$$

For $3 / 5<\lambda<1$, we have

$$
(1+t)^{\frac{5}{2}-\frac{3 \lambda}{2}}\left(\left\|V_{t}\right\|^{2}+\left\|V_{x}\right\|^{2}\right) \leq C\left(\left\|V_{0}\right\|_{1}^{2}+\left\|U_{0}\right\|^{2}+\delta\right),
$$

and

$$
\int_{0}^{t}(1+s)^{\beta-\lambda+1}\left\|V_{t}\right\|^{2} d s \leq C(1+t)^{\beta+\frac{3 \lambda}{2}-\frac{3}{2}}\left(\left\|V_{0}\right\|_{1}^{2}+\left\|U_{0}\right\|^{2}+\delta\right),
$$

for any $3 / 2-(3 \lambda) / 2<\beta<\lambda$.

Proof. Multiplying (117) by $(1+t)^{1-\lambda}$, for the case of $0 \leq \lambda<3 / 5$, and noting $\beta=\lambda$, we have

$$
\begin{aligned}
& \frac{1}{2} \frac{d}{d t} \int\left[(1+t)^{1+\lambda} V_{t}^{2}-(1+t)^{1+\lambda} p^{\prime}(\bar{v}) V_{x}^{2}\right] d x+C \int(1+t) V_{t}^{2} d x \\
& \leq \frac{d}{d t} \int(1+t)^{1+\lambda}\left(\int_{\bar{v}}^{V_{x}+\bar{v}+\vec{v}} p(s) d s-p(\bar{v}) V_{x}-\frac{1}{2} p^{\prime}(\bar{v}) V_{x}^{2}\right) d x \\
& \quad-(1-\lambda) \int(1+t)^{\lambda}\left(\int_{\bar{v}}^{V_{x}+\bar{v}+\vec{v}} p(s) d s-p(\bar{v}) V_{x}-\frac{1}{2} p^{\prime}(\bar{v}) V_{x}^{2}\right) d x \\
& \quad+C \int\left((1+t)^{\lambda} V_{t}^{2}-(1+t)^{\lambda} p^{\prime}(\bar{v}) V_{x}^{2}\right) d x \\
& \quad+C \int(1+t)^{\lambda} V_{x}^{2} d x+C \delta^{2}(1+t)^{(5 \lambda / 2)-(5 / 2)}+C \delta^{2}(1+t)^{-(\lambda / 2)-(3 / 2)} .
\end{aligned}
$$

Integrating the above inequality in $t$ over $(0, t)$, using (98), we get

$$
(1+t)^{\lambda+1}\left(\left\|V_{t}\right\|^{2}+\left\|V_{x}\right\|^{2}\right)+\int_{0}^{t}(1+s)\left\|V_{t}\right\|^{2} d s \leq C\left(\left\|V_{0}\right\|_{1}^{2}+\left\|U_{0}\right\|^{2}+\delta\right),
$$

for $0 \leq \lambda<3 / 5$.

This completes the proof of (128). For the case of $3 / 5<\lambda<1$, we can use the similar method to obtain (129) and (131).

Next, we will derive decay rates on the higher derivatives of the global solution $V(x, t)$.

Lemma 4.3. Under the assumptions of Theorem 2.4, if $\varepsilon$ is small enough, it holds that

$$
\begin{aligned}
(1+t)^{2 \lambda+2}\left(\left\|V_{x t}\right\|^{2}+\left\|V_{x x}\right\|^{2}\right) & \\
& +\int_{0}^{t}\left((1+s)^{\lambda+2}\left\|V_{x t}\right\|^{2}+(1+s)^{2 \lambda+1}\left\|V_{x x}\right\|^{2}\right) d s \\
\leq & C\left(\left\|V_{0}\right\|_{2}^{2}+\left\|U_{0}\right\|_{1}^{2}+\delta\right), \quad \text { for } \quad 0 \leq \lambda<\frac{3}{5} .
\end{aligned}
$$

For $3 / 5<\lambda<1$, we have

$$
(1+t)^{\frac{7}{2}-\frac{\lambda}{2}}\left(\left\|V_{x t}\right\|^{2}+\left\|V_{x x}\right\|^{2}\right) \leq C\left(\left\|V_{0}\right\|_{2}^{2}+\left\|U_{0}\right\|_{1}^{2}+\delta\right),
$$

and

$$
\begin{aligned}
& \int_{0}^{t}\left((1+s)^{\beta+\lambda+1}\left\|V_{x x}\right\|^{2}+(1+s)^{\beta+2}\left\|V_{x t}\right\|^{2}\right) d s \\
& \quad \leq C(1+t)^{\beta+(3 \lambda / 2)-(3 / 2)}\left(\left\|V_{0}\right\|_{2}^{2}+\left\|U_{0}\right\|^{1}+\delta\right),
\end{aligned}
$$

for any $3 / 2-(3 \lambda) / 2<\beta<\lambda$.

Proof. Multiplying (74) by $(1+t)^{\beta+\lambda} V_{x t}$ and integrating the resulting equation with respect to $x$ over $\mathbb{R}^{+}$, by using integrations by parts, we can get

$$
\begin{aligned}
& \frac{1}{2} \frac{d}{d t} \int\left[(1+t)^{\beta+\lambda} V_{x t}^{2}-(1+t)^{\beta+\lambda} p^{\prime}(\bar{v}) V_{x x}^{2}\right] d x \\
& \quad+\int \alpha(1+t)^{\beta} V_{x t}^{2} d x=\frac{\beta+\lambda}{2} \int(1+t)^{\beta+\lambda-1} V_{x t}^{2} d x \\
& \quad-\frac{\beta+\lambda}{2} \int(1+t)^{\beta+\lambda-1} p^{\prime}(\bar{v}) V_{x x}^{2} d x-\frac{1}{2} \int(1+t)^{\beta+\lambda} p^{\prime \prime}(\bar{v}) \bar{v}_{t} V_{x x}^{2} d x \\
& \quad-\int(1+t)^{\beta+\lambda} p^{\prime \prime \prime}(\bar{v})\left|\bar{v}_{x}\right|^{2} V_{x} V_{x t} d x-\int(1+t)^{\beta+\lambda} p^{\prime \prime}(\bar{v}) \bar{v}_{x x} V_{x} V_{x t} d x \\
& \quad-\int(1+t)^{\beta+\lambda} p^{\prime \prime}(\bar{v}) \bar{v}_{x} V_{x x} V_{x t} d x+\int(1+t)^{\beta+\lambda} F_{x} V_{x t} d x:=\sum_{k=1}^{7} L_{k} .
\end{aligned}
$$

By using the Cauchy-Schwarz's inequality, Lemma 2.2 and Corollary 2.3 to address the following estimates

$$
\begin{gathered}
L_{1}+L_{2} \leq C \int(1+t)^{\beta+\lambda-1}\left(V_{x t}^{2}+V_{x x}^{2}\right) d x, \\
L_{3} \leq C \delta \int(1+t)^{\beta+(\lambda / 2)-(3 / 2)} V_{x x}^{2} d x, \\
L_{4} \leq \frac{\alpha}{16} \int(1+t)^{\beta} V_{x t}^{2} d x+C \int(1+t)^{\beta+2 \lambda}\left|\bar{v}_{x}\right|^{4} V_{x}^{2} d x \\
\leq \frac{\alpha}{16} \int(1+t)^{\beta} V_{x t}^{2} d x+C \delta^{4} \int(1+t)^{\beta-2 \lambda-4} V_{x}^{2} d x, \\
L_{5} \leq \frac{\alpha}{16} \int(1+t)^{\beta} V_{x t}^{2} d x+C \int(1+t)^{\beta+2 \lambda}\left|\bar{v}_{x x}\right|^{2} V_{x}^{2} d x \\
\leq \frac{\alpha}{16} \int(1+t)^{\beta} V_{x t}^{2} d x+C \delta^{2} \int(1+t)^{\beta-\lambda-3} V_{x}^{2} d x,
\end{gathered}
$$


and

$$
\begin{aligned}
L_{6} & \leq \frac{\alpha}{16} \int(1+t)^{\beta} V_{x t}^{2} d x+C \int(1+t)^{\beta+2 \lambda}\left|\bar{v}_{x}\right|^{2} V_{x x}^{2} d x \\
& \leq \frac{\alpha}{16} \int(1+t)^{\beta} V_{x t}^{2} d x+C \delta^{2} \int(1+t)^{\beta-2} V_{x x}^{2} d x .
\end{aligned}
$$

Now, we turn to estimate $L_{7}$ as follows:

$$
\begin{aligned}
L_{7}=\int(1+t)^{\beta+\lambda} F_{x} V_{x t} d x= & \int\left[\frac{(1+t)^{\beta+2 \lambda}}{\alpha} p^{\prime}\left(v_{+}\right) \bar{v}_{x x t}+\frac{\lambda(1+t)^{\beta+2 \lambda-1}}{\alpha}\right. \\
& \cdot p^{\prime}\left(v_{+}\right) \bar{v}_{x x}-(1+t)^{\beta+\lambda}\left(p\left(V_{x}+\bar{v}+\widehat{v}\right)-p(\bar{v})-p^{\prime}(\bar{v}) V_{x}\right)_{x x} \\
& \left.+(1+t)^{\beta+\lambda}\left(\left(p^{\prime}\left(v_{+}\right)-p^{\prime}(\bar{v})\right) \bar{v}_{x}\right)_{x}\right] V_{x t} d x:=\sum_{k=1}^{4} M_{k} .
\end{aligned}
$$

By using Lemma 2.1, Lemma 2.2, Corollary 2.3 and a priori assumption (97), we have

$$
\begin{aligned}
M_{1} & \leq \frac{\alpha}{16} \int(1+t)^{\beta} V_{x t}^{2} d x+C \int(1+t)^{\beta+4 \lambda}\left|\bar{v}_{x x t}\right|^{2} d x \\
& \leq \frac{\alpha}{16} \int(1+t)^{\beta} V_{x t}^{2} d x+C \delta^{2}(1+t)^{\beta+(3 \lambda / 2)-(9 / 2)} \\
M_{2} & \leq \frac{\alpha}{16} \int(1+t)^{\beta} V_{x t}^{2} d x+C \int(1+t)^{\beta+4 \lambda-2}\left|\bar{v}_{x x}\right|^{2} d x \\
& \leq \frac{\alpha}{16} \int(1+t)^{\beta} V_{x t}^{2} d x+C \delta^{2}(1+t)^{\beta+(3 \lambda / 2)-(9 / 2)}
\end{aligned}
$$

$$
\begin{aligned}
\hline M_{3}= & \int(1+t)^{\beta+\lambda}\left(p\left(V_{x}+\bar{v}+\widehat{v}\right)-p(\bar{v})-p^{\prime}(\bar{v}) V_{x}\right)_{x} V_{x x t} d x \\
\leq & (1+t)^{\beta+\lambda} \frac{1}{2} \frac{d}{d t} \int\left(p^{\prime}\left(V_{x}+\bar{v}+\widehat{v}\right)-p^{\prime}(\bar{v})\right) V_{x x}^{2} d x-\frac{1}{2} \int(1+t)^{\beta+\lambda}\left(p^{\prime \prime}\left(V_{x}+\bar{v}+\widehat{v}\right)\left(V_{x t}+\bar{v}_{t}+\widehat{v}_{t}\right)-p^{\prime \prime}(\bar{v}) \bar{v}_{t}\right) V_{x x}^{2} d x \\
& +\int(1+t)^{\beta+\lambda}\left(p^{\prime}\left(V_{x}+\bar{v}+\widehat{v}\right)-p^{\prime}(\bar{v})-p^{\prime \prime}(\bar{v}) V_{x}\right) \bar{v}_{x} V_{x x t} d x+\int(1+t)^{\beta+\lambda} p^{\prime}\left(V_{x}+\bar{v}+\widehat{v}\right) \widehat{v}_{x} V_{x x t} d x \\
\leq & \frac{1}{2} \frac{d}{d t} \int(1+t)^{\beta+\lambda}\left(p^{\prime}\left(V_{x}+\bar{v}+\widehat{v}\right)-p^{\prime}(\bar{v})\right) V_{x x}^{2} d x-\frac{\beta+\lambda}{2} \int(1+t)^{\beta+\lambda-1}\left(p^{\prime}\left(V_{x}+\bar{v}+\widehat{v}\right)-p^{\prime}(\bar{v})\right) V_{x x}^{2} d x \\
& +C(\varepsilon+\delta) \int(1+t)^{\beta+\lambda-1} V_{x x}^{2} d x-\int(1+t)^{\beta+\lambda} \bar{v}_{x x} V_{x t}\left(p^{\prime}\left(V_{x}+\bar{v}+\widehat{v}\right)-p^{\prime}(\bar{v})-p^{\prime \prime}(\bar{v}) V_{x}\right) d x \\
& -\int(1+t)^{\beta+\lambda} \bar{v}_{x} V_{x t}\left(p^{\prime \prime}\left(V_{x}+\bar{v}+\widehat{v}\right)-p^{\prime \prime}(\bar{v})\right) V_{x x} d x-\int(1+t)^{\beta+\lambda} \bar{v}_{x}^{2} V_{x t}\left(p^{\prime \prime}\left(V_{x}+\bar{v}+\widehat{v}\right)-p^{\prime \prime}(\bar{v})-p^{\prime \prime \prime}(\bar{v}) V_{x}\right) d x \\
& -\int(1+t)^{\beta+\lambda} V_{x t} \bar{v}_{x} \widehat{v}_{x} p^{\prime \prime}\left(V_{x}+\bar{v}+\widehat{v}\right) d x-\int(1+t)^{\beta+\lambda} V_{x t} \widehat{v}_{x} p^{\prime \prime}\left(V_{x}+\bar{v}+\widehat{v}\right)\left(V_{x x}+\bar{v}+\widehat{v}\right) d x \\
& -\int(1+t)^{\beta+\lambda} V_{x t} \widehat{v}_{x x} p^{\prime}\left(V_{x}+\bar{v}+\widehat{v}\right) d x \\
\leq & \frac{1}{2} \frac{d}{d t} \int(1+t)^{\beta+\lambda}\left(p^{\prime}\left(V_{x}+\bar{v}+\widehat{v}\right)-p^{\prime}(\bar{v})\right) V_{x x}^{2} d x+\frac{\alpha}{16} \int(1+t)^{\beta} V_{x t}^{2} d x \\
& +C(\varepsilon+\delta) \int(1+t)^{\beta+\lambda-1} V_{x x}^{2} d x+C \delta^{2} \int(1+t)^{\beta-2} V_{x}^{2} d x+C \delta^{2}(1+t)^{\beta+(3 \lambda / 2)-(9 / 2)},
\end{aligned}
$$

and

$$
\begin{aligned}
M_{4} \leq & \frac{\alpha}{16} \int(1+t)^{\beta} V_{x t}^{2} d x \\
& +C \int(1+t)^{\beta+2 \lambda}\left(\left(p^{\prime}\left(v_{+}\right)-p^{\prime}(\bar{v})\right) \bar{v}_{x}\right)_{x}^{2} d x \\
\leq & \frac{\alpha}{16} \int(1+t)^{\beta} V_{x t}^{2} d x+C \delta^{2}(1+t)^{\beta-(3 \lambda / 2)-(7 / 2)} .
\end{aligned}
$$

Substituting (137)-(146) into (136), and noticing $\varepsilon, \delta$ sufficiently small, we have

$$
\begin{aligned}
& \frac{1}{2} \frac{d}{d t} \int\left[(1+t)^{\beta+\lambda} V_{x t}^{2}-(1+t)^{\beta+\lambda} p^{\prime}(\bar{v}) V_{x x}^{2}\right] d x \\
& \quad+\frac{\alpha}{2} \int(1+t)^{\beta} V_{x t}^{2} d x \\
& \leq \frac{1}{2} \frac{d}{d t} \int(1+t)^{\beta+\lambda}\left(p^{\prime}\left(V_{x}+\bar{v}+\widehat{v}\right)-p^{\prime}(\bar{v})\right) V_{x x}^{2} d x \\
& \quad+C \int(1+t)^{\beta+\lambda-1}\left(V_{x x}^{2}+V_{x t}^{2}\right) d x \\
& \quad+C \delta^{2} \int(1+t)^{\beta-2} V_{x}^{2} d x \\
& \quad+C \delta^{2}(1+t)^{\beta+(3 \lambda / 2)-(9 / 2)}+C \delta^{2}(1+t)^{\beta-(3 \lambda / 2)-(7 / 2)} .
\end{aligned}
$$

Multiplying (57) $)_{1}$ by $-(1+t)^{\beta} V_{x x}$ and integrating the resulting equality with respect to $x$ over $\mathbb{R}^{+}$, we have

$$
\begin{aligned}
& \frac{d}{d t} \int\left[(1+t)^{\beta} V_{x} V_{x t}+\frac{\alpha}{2}(1+t)^{\beta-\lambda} V_{x}^{2}\right] d x \\
& \quad+\frac{\alpha(\lambda-\beta)}{2} \int(1+t)^{\beta-\lambda-1} V_{x}^{2} d x-\int(1+t)^{\beta} p^{\prime}(\bar{v}) V_{x x}^{2} d x \\
& =\int(1+t)^{\beta} V_{x t}^{2} d x+\frac{\beta}{2} \frac{d}{d t} \int(1+t)^{\beta-1} V_{x}^{2} d x \\
& \quad+\frac{\beta(1-\beta)}{2} \int(1+t)^{\beta-2} V_{x}^{2} d x+\int(1+t)^{\beta} p^{\prime \prime}(\bar{v}) \bar{v}_{x} V_{x} V_{x x} d x \\
& \quad-\int(1+t)^{\beta} F V_{x x} d x .
\end{aligned}
$$

It is easy to see that

$$
\begin{aligned}
& \int(1+t)^{\beta} p^{\prime \prime}(\bar{v}) \bar{v}_{x} V_{x} V_{x x} d x \leq \frac{C_{0}}{10} \int(1+t)^{\beta} V_{x x}^{2} d x \\
& +C \int(1+t)^{\beta}\left|\bar{v}_{x}\right|^{2} V_{x}^{2} d x \leq \frac{C_{0}}{10} \int(1+t)^{\beta} V_{x x}^{2} d x \\
& +C \delta^{2} \int(1+t)^{\beta-2 \lambda-2} V_{x}^{2} d x .
\end{aligned}
$$




$$
\begin{aligned}
& \text { From (39), we have } \\
& \begin{array}{l}
-\int(1+t)^{\beta} F V_{x x} d x=-\int\left[\frac{(1+t)^{\beta+\lambda}}{\alpha} p^{\prime}\left(v_{+}\right) \bar{v}_{x t}\right. \\
\quad+\frac{\lambda(1+t)^{\beta+\lambda-1}}{\alpha} p^{\prime}\left(v_{+}\right) \bar{v}_{x}-(1+t)^{\beta}\left(p\left(V_{x}+\bar{v}+\widehat{v}\right)-p(\bar{v})-p^{\prime}(\bar{v}) V_{x}\right)_{x} \\
\left.\quad+(1+t)^{\beta}\left(p^{\prime}\left(v_{+}\right)-p^{\prime}(\bar{v})\right) \bar{v}_{x}\right] V_{x x} d x .
\end{array}
\end{aligned}
$$

By using Lemma 2.1, Lemma 2.2, Corollary 2.3 and a priori assumption (97), we have

$$
\begin{aligned}
&- \int \frac{(1+t)^{\beta+\lambda}}{\alpha} p^{\prime}\left(v_{+}\right) \bar{v}_{x t} V_{x x} d x \leq \frac{C_{0}}{10} \int(1+t)^{\beta} V_{x x}^{2} d x \\
&+C \int(1+t)^{\beta+2 \lambda}\left|\bar{v}_{x t}\right|^{2} d x \leq \frac{C_{0}}{10} \int(1+t)^{\beta} V_{x x}^{2} d x \\
&+C \delta^{2}(1+t)^{\beta+(\lambda / 2)-(7 / 2)}, \\
&-\int \frac{\lambda(1+t)^{\beta+\lambda-1}}{\alpha} p^{\prime}\left(v_{+}\right) \bar{v}_{x} V_{x x} d x \leq \frac{C_{0}}{10} \int(1+t)^{\beta} V_{x x}^{2} d x \\
&+C \int(1+t)^{\beta+2 \lambda-2}\left|\bar{v}_{x}\right|^{2} d x \leq \frac{C_{0}}{10} \int(1+t)^{\beta} V_{x x}^{2} d x \\
&+C \delta^{2}(1+t)^{\beta+(\lambda / 2)-(7 / 2)}, \\
&=t)^{\beta}\left(p\left(V_{x}+\bar{v}+\widehat{v}\right)-p(\bar{v})-p^{\prime}(\bar{v}) V_{x}\right)_{x} V_{x x} d x \\
&++t)^{\beta} \int\left(p^{\prime}\left(V_{x}+\bar{v}+\widehat{v}\right)-p^{\prime}(\bar{v})\right) V_{x x}^{2} d x \\
&+(1+t)^{\beta} \int\left(p^{\prime}\left(V_{x}+\bar{v}+\widehat{v}\right)-p^{\prime}(\bar{v})-p^{\prime \prime}(\bar{v}) V_{x}\right) \bar{v}_{x} V_{x x} d x \\
&+(1+t)^{\beta} \int p^{\prime}\left(V_{x}+\bar{v}+\widehat{v}\right) \widehat{v}_{x} V_{x x} d x \leq C \int(1+t)^{\beta}\left(\left|V_{x}\right|+|\widehat{v}|\right) V_{x x}^{2} d x \\
&+ \frac{C_{0}}{10} \int(1+t)^{\beta} V_{x x}^{2} d x+\int(1+t)^{\beta}\left|\bar{v}_{x}\right|^{2}\left(|\widehat{v}|^{2}+\left|V_{x}\right|^{4}\right) d x \\
&+ C \int(1+t)^{\beta}\left|\widehat{v}_{x}\right|^{2} d x \leq C(\varepsilon+\delta) \int(1+t)^{\beta} V_{x x}^{2} d x \frac{C_{0}}{10} \int(1+t)^{\beta} V_{x x}^{2} d x \\
&+ C \varepsilon^{2} \delta^{2} \int(1+t)^{\beta-\lambda-1} V_{x}^{2} d x+C \delta^{2}(1+t)^{\beta+(\lambda / 2)-(7 / 2)}, \\
& \int(153)
\end{aligned}
$$

and

$$
\begin{aligned}
\int & (1+t)^{\beta}\left(p^{\prime}\left(v_{+}\right)-p^{\prime}(\bar{v})\right) \bar{v}_{x} V_{x x} d x \\
\leq & \frac{C_{0}}{10} \int(1+t)^{\beta} V_{x x}^{2} d x \\
& +C \int(1+t)^{\beta}\left(\left(p^{\prime}\left(v_{+}\right)-p^{\prime}(\bar{v})\right) \bar{v}_{x}\right)^{2} d x \\
\leq & \frac{C_{0}}{10} \int(1+t)^{\beta} V_{x x}^{2} d x+C \delta^{2}(1+t)^{\beta-(5 \lambda / 2)-(5 / 2)} .
\end{aligned}
$$

Substituting (149)-(154) into (158), and using the smallness of $\delta, \varepsilon$, we have

$$
\begin{aligned}
\frac{d}{d t} \int & {\left[(1+t)^{\beta} V_{x} V_{x t}+\frac{\alpha}{2}(1+t)^{\beta-\lambda} V_{x}^{2}\right] d x } \\
& +\frac{\alpha(\lambda-\beta)}{2} \int(1+t)^{\beta-\lambda-1} V_{x}^{2} d x+\frac{C_{0}}{2} \int(1+t)^{\beta} V_{x x}^{2} d x \\
\leq & \int(1+t)^{\beta} V_{x t}^{2} d x+\frac{\beta}{2} \frac{d}{d t} \int(1+t)^{\beta-1} V_{x}^{2} d x \\
& +\frac{\beta(1-\beta)}{2} \int(1+t)^{\beta-2} V_{x}^{2} d x+C \delta^{2} \int(1+t)^{\beta-\lambda-1} V_{x}^{2} d x \\
& +C \delta^{2}(1+t)^{\beta+(\lambda / 2)-(7 / 2)}+C \delta^{2}(1+t)^{\beta-(5 \lambda / 2)-(5 / 2)} .
\end{aligned}
$$

Multiplying (147) by $h$ and adding up the resulting inequality and (155), we get

$$
\begin{aligned}
& \frac{d}{d t} \int\left[(1+t)^{\beta} V_{x} V_{x t}+\frac{\alpha}{2}(1+t)^{\beta-\lambda} V_{x}^{2}\right. \\
& \left.+\frac{h}{2}(1+t)^{\beta+\lambda} V_{x t}^{2}-\frac{h}{2}(1+t)^{\beta+\lambda} p^{\prime}(\bar{v}) V_{x x}^{2}\right] d x \\
& \quad+\frac{\alpha(\lambda-\beta)}{2} \int(1+t)^{\beta-\lambda-1} V_{x}^{2} d x+\frac{C_{0}}{2} \int(1+t)^{\beta} V_{x x}^{2} d x \\
& \quad+\left(\frac{\alpha h}{2}-1\right) \int(1+t)^{\beta} V_{x t}^{2} d x \\
& \leq \frac{h}{2} \frac{d}{d t} \int(1+t)^{\beta+\lambda}\left(p^{\prime}\left(V_{x}+\bar{v}+\widehat{v}\right)-p^{\prime}(\bar{v})\right) V_{x x}^{2} d x \\
& \quad+\frac{\beta}{2} \frac{d}{d t} \int(1+t)^{\beta-1} V_{x}^{2} d x+C \int(1+t)^{\beta-\lambda-1} V_{x}^{2} d x \\
& \quad+C h \int(1+t)^{\beta+\lambda-1}\left(V_{x x}^{2}+V_{x t}^{2}\right) d x \\
& \quad+C \delta^{2}(1+t)^{\beta+(\lambda / 2)-(7 / 2)}+C \delta^{2}(1+t)^{\beta-(5 \lambda / 2)-(5 / 2)} .
\end{aligned}
$$

Multiplying (157) by $(1+t)^{\lambda+1}$, we have

$$
\begin{aligned}
\frac{d}{d t} \int[ & (1+t)^{\beta+\lambda+1} V_{x} V_{x t}+\frac{\alpha}{2}(1+t)^{\beta+1} V_{x}^{2} \\
& \left.+\frac{h}{2}(1+t)^{\beta+2 \lambda+1} V_{x t}^{2}-\frac{h}{2}(1+t)^{\beta+2 \lambda+1} p^{\prime}(\bar{v}) V_{x x}^{2}\right] d x \\
& +\frac{\alpha(\lambda-\beta)}{2} \int(1+t)^{\beta} V_{x}^{2} d x+\frac{C_{0}}{2} \int(1+t)^{\beta+\lambda+1} V_{x x}^{2} d x \\
& +\left(\frac{\alpha h}{2}-1\right) \int(1+t)^{\beta+\lambda+1} V_{x t}^{2} d x \\
\leq & \frac{h}{2} \frac{d}{d t} \int(1+t)^{\beta+2 \lambda+1}\left(p^{\prime}\left(V_{x}+\bar{v}+\widehat{v}\right)-p^{\prime}(\bar{v})\right) V_{x x}^{2} d x \\
& +\frac{\beta}{2} \frac{d}{d t} \int(1+t)^{\beta+\lambda} V_{x}^{2} d x+C \int(1+t)^{\beta} V_{x}^{2} d x \\
& +C h \int(1+t)^{\beta+2 \lambda}\left(V_{x x}^{2}+V_{x t}^{2}\right) d x+C \delta^{2}(1+t)^{\beta+(3 \lambda / 2)-(5 / 2)} \\
& +C \delta^{2}(1+t)^{\beta-(3 \lambda / 2)-(3 / 2)}+C \int(1+t)^{\beta+2 \lambda}\left(p^{\prime}\left(V_{x}+\bar{v}+\widehat{v}\right)-p^{\prime}(\bar{v})\right) V_{x x}^{2} d x .
\end{aligned}
$$

Case $1(0 \leq \lambda<3 / 5) . \quad \beta=\lambda, h=6 / \alpha$. There exists constant $T_{2}>0$, such that for any $t \geq T_{2}$, it holds $C h(1+t)^{\lambda-1} \leq \min \left\{C_{0} / 4,1 / 2(\alpha h / 2-1)\right\}$. Therefore, if $t \geq T_{2}$, from (157), we have

$$
\begin{aligned}
\frac{d}{d t} H_{2}(t)+C \int(1+t)^{2 \lambda+1}\left(V_{x x}^{2}+V_{x t}^{2}\right) d x \\
\leq \frac{3}{\alpha} \frac{d}{d t} \int(1+t)^{3 \lambda+1}\left(p^{\prime}\left(V_{x}+\bar{v}+\widehat{v}\right)-p^{\prime}(\bar{v})\right) V_{x x}^{2} d x \\
\quad+\frac{\lambda}{2} \frac{d}{d t} \int(1+t)^{2 \lambda} V_{x}^{2} d x+C \int(1+t)^{\lambda} V_{x}^{2} d x \\
\quad+C \delta^{2}(1+t)^{(5 \lambda / 2)-(5 / 2)}+C \delta^{2}(1+t)^{-(\lambda / 2)-(3 / 2)}
\end{aligned}
$$

where

$$
H_{2}(t) \sim(1+t)^{\lambda+1}\left\|V_{x}\right\|^{2}+(1+t)^{3 \lambda+1}\left\|V_{x x}\right\|^{2}+(1+t)^{3 \lambda+1}\left\|V_{x t}\right\|^{2} .
$$

Integrating (158) on $\left[T_{2}, t\right]$ with respect to the time $t$ and using (98), (126), $0 \leq \lambda<3 / 5$, we have 


$$
\begin{aligned}
& (1+t)^{3 \lambda+1}\left(\left\|V_{x x}\right\|^{2}+\left\|V_{x t}\right\|^{2}\right)+\int_{T_{2}}^{t}(1+s)^{2 \lambda+1}\left(\left\|V_{x x}\right\|^{2}+\left\|V_{x t}\right\|^{2}\right) d s \\
& \leq C\left(H_{2}\left(T_{2}\right)+\left\|V_{0}\right\|_{1}^{2}+\left\|U_{0}\right\|^{2}+\delta\right) .
\end{aligned}
$$

From (160), (53) and (54), we have for any $t>0$

$$
\begin{aligned}
& (1+t)^{3 \lambda+1}\left(\left\|V_{x x}\right\|^{2}+\left\|V_{x t}\right\|^{2}\right)+\int_{0}^{t}(1+s)^{2 \lambda+1}\left(\left\|V_{x x}\right\|^{2}+\left\|V_{x t}\right\|^{2}\right) d s \\
& \quad \leq C\left(\left\|V_{0}\right\|_{2}^{2}+\left\|U_{0}\right\|_{1}^{2}+\delta\right) .
\end{aligned}
$$

Multiply (147) by $(1+t)^{2}$ with $\beta=\lambda$, we have

$$
\begin{aligned}
& \frac{1}{2} \frac{d}{d t} \int\left[(1+t)^{2+2 \lambda} V_{x t}^{2}-(1+t)^{2+2 \lambda} p^{\prime}(\bar{v}) V_{x x}^{2}\right] d x \\
& \quad+\frac{\alpha}{2} \int(1+t)^{\lambda+2} V_{x t}^{2} d x \leq \frac{1}{2} \frac{d}{d t} \int(1+t)^{2+2 \lambda}\left(p^{\prime}\left(V_{x}+\bar{v}+\widehat{v}\right)-p^{\prime}(\bar{v})\right) V_{x x}^{2} d x \\
& \quad+C \int(1+t)^{2 \lambda+1}\left(V_{x x}^{2}+V_{x t}^{2}\right) d x+C \delta^{2} \int(1+t)^{\lambda} V_{x}^{2} d x \\
& \quad+C \delta^{2}(1+t)^{(5 \lambda / 2)-(5 / 2)}+C \delta(1+t)^{-(\lambda / 2)-(3 / 2)} .
\end{aligned}
$$

Integrating (162) on $(0, t)$, using (98), (161) and $0 \leq \lambda<3 / 5$, we have (133).

Case $2(3 / 5<\lambda<1)$. In this case, we take $3 / 2-3 \lambda / 2<\beta<\lambda$. There exists constant $T_{3}>0$, such that for any $t \geq T_{3}$, it holds $C h(1+t)^{\lambda-1} \leq \min \left\{C_{0} / 4,1 / 2((\alpha h) / 2-1)\right\}$, where we choose $h=6 / \alpha$. Therefore if $t \geq T_{3}$, from (157), we have

$$
\begin{aligned}
& \frac{d}{d t} H_{3}(t)+C \int(1+t)^{\beta+\lambda+1}\left(V_{x x}^{2}+V_{x t}^{2}\right) d x+C \int(1+t)^{\beta} V_{x}^{2} d x \\
& \leq \frac{h}{2} \frac{d}{d t} \int(1+t)^{\beta+2 \lambda+1}\left(p^{\prime}\left(V_{x}+\bar{v}+\widehat{v}\right)-p^{\prime}(\bar{v})\right) V_{x x}^{2} d x \\
&+\frac{\beta}{2} \frac{d}{d t} \int(1+t)^{\beta+\lambda} V_{x}^{2} d x+C \int(1+t)^{\beta} V_{x}^{2} d x \\
&+C \delta^{2}(1+t)^{\beta+(3 \lambda / 2)-(5 / 2)}
\end{aligned}
$$

where

$$
H_{3}(t) \sim(1+t)^{\beta+1}\left\|V_{x}\right\|^{2}+(1+t)^{\beta+2 \lambda+1}\left(\left\|V_{x x}\right\|^{2}+\left\|V_{x t}\right\|^{2}\right) .
$$

Integrating (163) on $\left[T_{3}, t\right]$ with respect to the time $t$ over $\left[T_{3}, t\right]$ and using (98), (128), $3 / 5<\lambda<1$, we have

$$
\begin{aligned}
& H_{3}(t)+\int_{T_{3}}^{t}(1+t)^{\beta+\lambda+1}\left(\left\|V_{x x}\right\|^{2}+\left\|V_{x t}\right\|^{2}\right) d s \\
& \quad \leq H_{3}\left(T_{3}\right)+C(1+t)^{\beta+(3 \lambda / 2)-(3 / 2)}\left(\left\|V_{0}\right\|_{1}^{2}+\left\|U_{0}\right\|^{2}+\delta\right),
\end{aligned}
$$

which, together with (51), (52), and $\beta+3 \lambda / 2-3 / 2>0$, yields

$$
\begin{aligned}
& (1+t)^{\beta+2 \lambda+1}\left(\left\|V_{x x}\right\|^{2}+\left\|V_{x x}\right\|^{2}\right)+\int_{0}^{t}(1+s)^{\beta+\lambda+1}\left(\left\|V_{x x}\right\|^{2}+\left\|V_{x t}\right\|^{2}\right) d s \\
& \leq C(1+t)^{\beta+(3 \lambda / 2)-(3 / 2)}\left(\left\|V_{0}\right\|_{2}^{2}+\left\|U_{0}\right\|_{1}^{2}+\delta\right) .
\end{aligned}
$$

Finally, multiplying $(147)$ by $(1+t)^{2}$, we have

$$
\begin{aligned}
& \frac{1}{2} \frac{d}{d t} \int\left[(1+t)^{\beta+\lambda+2} V_{x t}^{2}-(1+t)^{\beta+\lambda+2} p^{\prime}(\bar{v}) V_{x x}^{2}\right] d x \\
& \quad+\frac{\alpha}{2} \int(1+t)^{\beta+2} V_{x t}^{2} d x \leq \frac{1}{2} \frac{d}{d t} \int(1+t)^{\beta+\lambda+2}\left(p^{\prime}\left(V_{x}+\bar{v}+\widehat{v}\right)-p^{\prime}(\bar{v})\right) V_{x x}^{2} d x \\
& \quad+C \int(1+t)^{\beta+\lambda+1}\left(V_{x x}^{2}+V_{x t}^{2}\right) d x+C \int(1+t)^{\beta} V_{x}^{2} d x \\
& \quad+C \delta^{2}(1+t)^{\beta+(3 \lambda / 2)-(5 / 2)}+C \delta(1+t)^{\beta-(3 \lambda / 2)-(3 / 2)} .
\end{aligned}
$$

Integrating the above inequality over $(0, t)$ and using (99), (100), and (166), we get

$$
\begin{gathered}
(1+t)^{\beta+\lambda+2}\left(\left\|V_{x x}\right\|^{2}+\left\|V_{x t}\right\|^{2}\right)+\int_{0}^{t}(1+s)^{\beta+2}\left\|V_{x t}\right\|^{2} d s \\
\leq C(1+t)^{\beta+\frac{3 \lambda}{2}-\frac{3}{2}}\left(\left\|V_{0}\right\|_{2}^{2}+\left\|U_{0}\right\|_{1}^{2}+\delta\right),
\end{gathered}
$$

which combining with (166) gives (134) and (135). This completes the proof of Lemma 4.3.

Similar calculations to Lemma 4.3 yield the following lemma, the proof of which is omitted.

Lemma 4.4. Under the assumptions of Theorem 2.4, if $\varepsilon$ is small enough, it holds that

$$
\begin{gathered}
(1+t)^{3 \lambda+3}\left(\left\|V_{x x t}\right\|^{2}+\left\|V_{x x x}\right\|^{2}\right) \\
+\int_{0}^{t}\left((1+s)^{3+2 \lambda}\left\|V_{x x t}\right\|^{2}+(1+s)^{3 \lambda+2}\left\|V_{x x x}\right\|^{2}\right) d s \\
\leq C\left(\left\|V_{0}\right\|_{3}^{2}+\left\|U_{0}\right\|_{2}^{2}+\delta\right), \quad \text { for } 0 \leq \lambda<\frac{3}{5} .
\end{gathered}
$$

For $3 / 5<\lambda<1$, we have

$$
(1+t)^{(9 / 2)+(\lambda / 2)}\left(\left\|V_{x x t}\right\|^{2}+\left\|V_{x x x}\right\|^{2}\right) \leq C\left(\left\|V_{0}\right\|_{3}^{2}+\left\|U_{0}\right\|_{2}^{2}+\delta\right)
$$

and

$$
\begin{gathered}
\int_{0}^{t}\left((1+s)^{\beta+2 \lambda+2}\left\|V_{x x x}\right\|^{2}+(1+s)^{\beta+\lambda+3}\left\|V_{x x t}\right\|^{2}\right) d s \\
\leq C(1+t)^{\beta+(3 \lambda / 2)-(3 / 2)}\left(\left\|V_{0}\right\|_{3}^{2}+\left\|U_{0}\right\|_{2}^{2}+\delta\right),
\end{gathered}
$$

for any $3 / 2-3 \lambda / 2<\beta<\lambda$.

With Lemmas 4.1-4.4 in hand, by the Sobolev inequality, it is easy to know that if $0 \leq \lambda<3 / 5$,

$$
\left\|V_{x}(\cdot, t)\right\|_{L^{\infty}} \leq C(1+t)^{-(3 / 4)(\lambda+1)}\left(\left\|V_{0}\right\|_{3}+\left\|U_{0}\right\|_{2}+\delta^{1 / 2}\right) \leq \frac{\varepsilon}{2},
$$

$\left\|V_{x t}(\cdot, t)\right\|_{L^{\infty}} \leq C(1+t)^{-(5 / 4)(\lambda+1)}\left(\left\|V_{0}\right\|_{3}+\left\|U_{0}\right\|_{2}+\delta^{1 / 2}\right) \leq \frac{\varepsilon}{2}(1+t)^{-1}$,

and

$$
\begin{aligned}
\left\|V_{x x}(\cdot, t)\right\|_{L^{\infty}} & \leq C(1+t)^{-(5 / 4)(\lambda+1)}\left(\left\|V_{0}\right\|_{3}+\left\|U_{0}\right\|_{2}+\delta^{1 / 2}\right) \\
& \leq \frac{\varepsilon}{2}(1+t)^{-(\lambda+1 / 2)} .
\end{aligned}
$$

And if $3 / 5<\lambda<1$, we get

$\left\|V_{x}(\cdot, t)\right\|_{L^{\infty}} \leq C(1+t)^{-(3-\lambda / 2)}\left(\left\|V_{0}\right\|_{3}+\left\|U_{0}\right\|_{2}+\delta^{1 / 2}\right) \leq \frac{\varepsilon}{2}$, 
$\left\|V_{x t}(\cdot, t)\right\|_{L^{\infty}} \leq C(1+t)^{-2}\left(\left\|V_{0}\right\|_{3}+\left\|U_{0}\right\|_{2}+\delta^{1 / 2}\right) \leq \frac{\varepsilon}{2}(1+t)^{-1}$,

and

$$
\left\|V_{x x}(\cdot, t)\right\|_{L^{\infty}} \leq C(1+t)^{-2}\left(\left\|V_{0}\right\|_{3}+\left\|U_{0}\right\|_{2}+\delta^{1 / 2}\right) \leq \frac{\varepsilon}{2}(1+t)^{-(\lambda+1 / 2)} .
$$

provided $\left\|V_{0}\right\|_{3}+\left\|U_{0}\right\|_{2}+\delta \leq 1$. Up to now, we thus close $a$ priori assumption (97) about $\left(V_{x}, V_{x t}, V_{x x}\right)$ from Lemmas 4.1-4.4.

For the decay rates of $U=V_{t}$, we give the following two lemmas.

Lemma 4.5. Under the assumptions of Theorem 2.4, if $\varepsilon$ is small enough, it holds that

$$
\begin{aligned}
(1+t)^{2}\|U\|^{2} & +(1+t)^{\lambda+3}\left(\left\|U_{t}\right\|^{2}+\left\|U_{x}\right\|^{2}\right) \\
& +\int_{0}^{t}\left((1+s)^{\lambda+2}\left\|U_{x}\right\|^{2}+(1+s)^{3}\left\|U_{t}\right\|^{2}\right) d s \\
& \leq C\left(\left\|V_{0}\right\|_{2}^{2}+\left\|U_{0}\right\|_{1}^{2}+\delta\right), \quad \text { for } 0 \leq \lambda<\frac{3}{5} .
\end{aligned}
$$

For $3 / 5<\lambda<1$, we have

$$
\begin{aligned}
& (1+t)^{(7 / 2)-(5 \lambda / 2)}\|U\|^{2}+(1+t)^{(9 / 2)-(3 \lambda / 2)}\left(\left\|U_{t}\right\|^{2}+\left\|U_{x}\right\|^{2}\right) \\
& \leq C\left(\left\|V_{0}\right\|_{2}^{2}+\left\|U_{0}\right\|_{1}^{2}+\delta\right),
\end{aligned}
$$

and

$$
\begin{aligned}
& \int_{0}^{t}\left[(1+s)^{\beta+2}\left\|U_{x}\right\|^{2}+(1+s)^{\beta-\lambda+3}\left\|U_{t}\right\|^{2}\right] d s \\
& \quad \leq C(1+t)^{\beta+(3 \lambda / 2)-(3 / 2)}\left(\left\|V_{0}\right\|_{2}^{2}+\left\|U_{0}\right\|_{1}^{2}+\delta\right),
\end{aligned}
$$

for any $(3 / 2)-(3 \lambda / 2)<\beta<\lambda$.

Proof. Differentiating (57) ${ }_{1}$ with respect to $t$, we get

$$
U_{t t}+\left(p^{\prime}(\bar{v}) V_{x}\right)_{x t}+\frac{\alpha}{(1+t)^{\lambda}} U_{t}=\frac{\alpha \lambda}{(1+t)^{\lambda+1}} U+F_{t} .
$$

Multiplying (181) by $(1+t)^{\beta+\lambda} U_{t}$, and integrating the resulting equality in $x$ over $\mathbb{R}^{+}$, and after tedious calculations, we have

$$
\begin{aligned}
\frac{1}{2} \frac{d}{d t} \int\left[(1+t)^{\beta+\lambda} U_{t}^{2}-(1+t)^{\beta+\lambda} p^{\prime}(\bar{v}) U_{x}^{2}\right] d x+\frac{\alpha}{2} \int(1+t)^{\beta} U_{t}^{2} d x \\
\leq \frac{1}{2} \frac{d}{d t} \int(1+t)^{\beta+\lambda}\left(p^{\prime}\left(V_{x}+\bar{v}+\widehat{v}\right)-p^{\prime}(\bar{v})\right) U_{x}^{2} d x+C \int(1+t)^{\beta-2} U^{2} d x \\
\quad+C \int(1+t)^{\beta+\lambda-1} U_{x}^{2} d x+\frac{\beta+\lambda}{2} \int(1+t)^{\beta+\lambda-1} U_{t}^{2} d x \\
\quad+C \delta^{2} \int(1+t)^{\beta+\lambda-3} V_{x}^{2} d x \\
\quad+C \delta^{2} \int(1+t)^{\beta+2 \lambda-2} V_{x x}^{2} d x+C \delta(1+t)^{\beta+(5 \lambda / 2)-(11 / 2)} .
\end{aligned}
$$

Similarly, multiplying (181) by $(1+t)^{\beta} U$, from Lemma 4.2 and after complicated calculations, we have

$$
\begin{aligned}
& \frac{d}{d t} \int\left[(1+t)^{\beta} U U_{t}+\frac{\alpha}{2}(1+t)^{\beta-\lambda} U^{2}\right] d x \\
& \quad+\frac{\alpha(\lambda-\beta)}{2} \int(1+t)^{\beta-\lambda-1} U^{2} d x+\frac{C_{0}}{2} \int(1+t)^{\beta} U_{x}^{2} d x \\
& \leq \int(1+t)^{\beta} U_{t}^{2} d x+\frac{\beta}{2} \frac{d}{d t} \int(1+t)^{\beta-1} U^{2} d x \\
& \quad+\frac{\beta(1-\beta)}{2} \int(1+t)^{\beta-\lambda-1} U^{2} d x \\
& \quad+C \delta^{2} \int(1+t)^{\beta-2} V_{x}^{2} d x+C \delta^{2}(1+t)^{\beta+(3 \lambda / 2)-(9 / 2)}
\end{aligned}
$$

Multiplying (172) by $h$ and adding up the resulting inequality and (173), we get

$$
\begin{aligned}
& \frac{d}{d t} \int\left[(1+t)^{\beta} U U_{t}+\frac{\alpha}{2}(1+t)^{\beta-\lambda} U^{2}+\frac{h}{2}(1+t)^{\beta+\lambda} U_{t}^{2}\right. \\
& \left.\quad-\frac{h}{2}(1+t)^{\beta+\lambda} p^{\prime}(\bar{v}) U_{x}^{2}\right] d x \\
& +\frac{\alpha(\lambda-\beta)}{2} \int(1+t)^{\beta-\lambda-1} U^{2} d x+\frac{C_{0}}{2} \int(1+t)^{\beta} U_{x}^{2} d x \\
& +\left(\frac{\alpha h}{2}-1\right) \int(1+t)^{\beta} U_{t}^{2} d x \leq \frac{h}{2} \frac{d}{d t} \int(1+t)^{\beta+\lambda} \\
& \quad \cdot\left(p^{\prime}\left(V_{x}+\bar{v}+\widehat{v}\right)-p^{\prime}(\bar{v})\right) U_{x}^{2} d x+\frac{\beta}{2} \frac{d}{d t} \int(1+t)^{\beta-1} U^{2} d x \\
& +C \int(1+t)^{\beta-\lambda-1} U^{2} d x+C \int(1+t)^{\beta+\lambda-1}\left(U_{x}^{2}+U_{t}^{2}\right) d x \\
& +C \delta^{2} \int(1+t)^{\beta-2} V_{x}^{2} d x+C \delta^{2} \int(1+t)^{\beta+2 \lambda-2} V_{x x}^{2} d x \\
& +C \delta^{2}(1+t)^{\beta+(3 \lambda / 2)-(9 / 2) .}
\end{aligned}
$$

Case $1(0 \leq \lambda<3 / 5)$. In this case, we take $\beta=\lambda, h=6 / \alpha$. Multiplying (184) by $(1+t)^{1-\lambda},(1+t)^{2-2 \lambda},(1+t)^{2}$ respectively, then integrating the resulting equations and (4.88) with respect to $t$ over $[0, t]$ respectively, we have

$$
\begin{aligned}
(1+t)^{2}\|U\|^{2}+ & (1+t)^{2 \lambda+2}\left(\left\|U_{t}\right\|^{2}+\left\|U_{x}\right\|^{2}\right) \\
& +\int_{0}^{t}(1+s)^{\lambda+2}\left(\left\|U_{x}\right\|^{2}+\left\|U_{t}\right\|^{2}\right) d s \\
\leq & C\left(\left\|V_{0}\right\|_{2}^{2}+\left\|U_{0}\right\|_{1}^{2}+\delta\right) .
\end{aligned}
$$

Multiplying (182) by $(1+t)^{3-\lambda}$, and integrating the resulting equation with respect to $t$ over $[0, t]$, we have

$$
\begin{aligned}
& (1+t)^{\lambda+3}\left(\left\|U_{t}\right\|^{2}+\left\|U_{x}\right\|^{2}\right)+\int_{0}^{t}(1+s)^{3}\left\|U_{t}\right\|^{2} d s \\
& \leq C\left(\left\|V_{0}\right\|_{2}^{2}+\left\|U_{0}\right\|_{1}^{2}+\delta\right) .
\end{aligned}
$$

Combining (183) and (184), we get (178).

Case $2(3 / 5<\lambda<1)$. In this case, we take $3 / 2-3 \lambda / 2<\beta<\lambda$, $h=6 / \alpha$. There exists constant $T_{5}>0$, such that for any $t \geq T_{5}$, it holds $C(1+t)^{\lambda-1} \leq \min \left\{C_{0} / 4,1 / 2((\alpha h / 2)-1)\right\}$. Multiplying (184) by $(1+t)^{2}$, we have 


$$
\begin{aligned}
\frac{d}{d t} \int[ & {[1+t)^{\beta+2} U U_{t}+\frac{\alpha}{2}(1+t)^{\beta-\lambda+2} U^{2}+\frac{h}{2}(1+t)^{\beta+\lambda+2} U_{t}^{2} } \\
& \left.-\frac{h}{2}(1+t)^{\beta+\lambda+2} p^{\prime}(\bar{v}) U_{x}^{2}\right] d x \\
& +\frac{C_{0}}{2} \int(1+t)^{\beta+2} U_{x}^{2} d x+\left(\frac{\alpha h}{2}-1\right) \int(1+t)^{\beta+2} U_{t}^{2} d x \\
\leq & \frac{h}{2} \frac{d}{d t} \int(1+t)^{\beta+\lambda+2}\left(p^{\prime}\left(V_{x}+\bar{v}+\widehat{v}\right)-p^{\prime}(\bar{v})\right) U_{x}^{2} d x \\
& +\frac{\beta}{2} \frac{d}{d t} \int(1+t)^{\beta+1} U^{2} d x+C \int(1+t)^{\beta-\lambda+1} U^{2} d x \\
& +C \int(1+t)^{\beta+\lambda+1}\left(U_{x}^{2}+U_{t}^{2}\right) d x+C \delta^{2} \int(1+t)^{\beta} V_{x}^{2} d x \\
& +C \delta^{2} \int(1+t)^{\beta+2 \lambda} V_{x x}^{2} d x+C \delta^{2}(1+t)^{\beta+(3 \lambda / 2)-(5 / 2)} .
\end{aligned}
$$

If $t \geq T_{5}$, integrating (187) over $\left(T_{5}, t\right)$, using (99), (100), (127), (128), (132) and (135), we get

$$
\begin{aligned}
H_{5}(t) & +\int_{T_{5}}^{t}(1+s)^{\beta+2}\left(\left\|U_{x}\right\|^{2}+\left\|U_{t}\right\|^{2}\right) d s \\
& \leq H_{5}\left(T_{5}\right)+C(1+t)^{\beta+(3 \lambda / 2)-(3 / 2)}\left(\left\|V_{0}\right\|_{2}^{2}+\left\|U_{0}\right\|_{1}^{2}+\delta\right),
\end{aligned}
$$

where

$$
H_{5}(t) \sim(1+t)^{\beta-\lambda+2}\|U\|^{2}+(1+t)^{\beta+\lambda+2}\left(\left\|U_{x}\right\|^{2}+\left\|U_{t}\right\|^{2}\right) .
$$

Next, if $0<t \leq T_{5}$, from (187), we know

$$
\begin{aligned}
\frac{d}{d t} H_{5}(t)+ & C \int(1+t)^{\beta+2} U_{x}^{2} d x+C \int(1+t)^{\beta+2} U_{t}^{2} d x \\
\leq & \frac{d}{d t} \int(1+t)^{\beta+\lambda+2}\left(p^{\prime}\left(V_{x}+\bar{v}+\widehat{v}\right)-p^{\prime}(\bar{v})\right) U_{x}^{2} d x \\
& +C(1+t)^{-1} H_{5}(t)+C \delta^{2} \int(1+t)^{\beta} V_{x}^{2} d x \\
& +C \delta^{2} \int(1+t)^{\beta+2 \lambda} V_{x x}^{2} d x+C \delta^{2}(1+t)^{\beta+(3 \lambda / 2)-(5 / 2)}
\end{aligned}
$$

Then using Gronwall's inequality, (99), (100), (134), and (135), and noting that $\beta+3 \lambda / 2>3 / 2$, we get

$$
\begin{aligned}
H_{5}(t)+ & \int_{0}^{t}(1+s)^{\beta+2}\left(\left\|U_{x}\right\|^{2}+\left\|U_{t}\right\|^{2}\right) d s \\
& \leq C(1+t)^{\beta+(3 \lambda / 2)-(3 / 2)}\left(\left\|V_{0}\right\|_{2}^{2}+\left\|U_{0}\right\|_{1}^{2}+\delta\right), \text { for any } t \in\left(0, T_{5}\right] .
\end{aligned}
$$

From (191) and (188), we have

$$
\begin{aligned}
H_{5}(t) & +\int_{0}^{t}(1+s)^{\beta+2}\left(\left\|U_{x}\right\|^{2}+\left\|U_{t}\right\|^{2}\right) d s \\
& \leq C(1+t)^{\beta+(3 \lambda / 2)-(3 / 2)}\left(\left\|V_{0}\right\|_{2}^{2}+\left\|U_{0}\right\|_{1}^{2}+\delta\right), \quad \text { for any } t \in(0, \infty) .
\end{aligned}
$$

Multiplying (182) by $(1+t)^{3-\lambda}$, and integrating the resulting equation with respect to $t$ over $[0, t]$, we have

$$
\begin{gathered}
(1+t)^{\beta+3}\left(\left\|U_{x}\right\|^{2}+\left\|U_{t}\right\|^{2}\right)+\int_{0}^{t}(1+s)^{\beta-\lambda+3}\left\|U_{t}\right\|^{2} d s \\
\leq C(1+t)^{\beta+(3 \lambda / 2)-(3 / 2)}\left(\left\|V_{0}\right\|_{2}^{2}+\left\|U_{0}\right\|_{1}^{2}+\delta\right) .
\end{gathered}
$$

Combining (192) and (193), we get (179) and (180). Therefore we completed the proof of Lemma 4.5.

Similar to the proof of Lemma 4.5, we can get the following estimates.

Lemma 4.6. Under the assumptions of Theorem 2.4, if $\varepsilon, \delta$ are small enough, it holds that

$$
\begin{gathered}
(1+t)^{2 \lambda+4}\left(\left\|U_{x t}\right\|^{2}+\left\|U_{x x}\right\|^{2}\right)+\int_{0}^{t}\left((1+s)^{2 \lambda+3}\left\|U_{x x}\right\|^{2}+(1+s)^{\lambda+4}\left\|U_{x t}\right\|^{2}\right) d s \\
\leq C\left(\left\|V_{0}\right\|_{3}^{2}+\left\|U_{0}\right\|_{2}^{2}+\delta\right), \quad \text { for } 0 \leq \lambda<\frac{3}{5} .
\end{gathered}
$$

For $3 / 5<\lambda<1$, we have

$$
(1+t)^{(11 / 2)-(\lambda / 2)}\left(\left\|U_{x t}\right\|^{2}+\left\|U_{x x}\right\|^{2}\right) \leq C\left(\left\|V_{0}\right\|_{3}^{2}+\left\|U_{0}\right\|_{2}^{2}+\delta\right),
$$

and

$$
\begin{aligned}
& \int_{0}^{t}\left[(1+s)^{\beta+\lambda+3}\left\|U_{x x}\right\|^{2}+(1+s)^{\beta+4}\left\|U_{x t}\right\|^{2}\right] d s \\
& \leq C(1+t)^{\beta-(3 / 2)+(3 \lambda / 2)}\left(\left\|V_{0}\right\|_{3}^{2}+\left\|U_{0}\right\|_{2}^{2}+\delta\right),
\end{aligned}
$$

for any $3 / 2-3 \lambda / 2<\beta<\lambda$.

Recalling Lemmas 4.1-4.6, we completed the desired decay estimates (42), (43), and (44).

By taking $\beta=\lambda=1 / 7, v=1$ and $\kappa=1$ in Lemmas 4.1-4.6, Theorem 2.6 can be shown by slightly modifying the proof of Theorem 2.4, and therefore, we omit its proof here.

\section{Data Availability}

No data were used to support this study.

\section{Conflicts of Interest}

The authors declare that they have no conflicts of interest.

\section{Acknowledgments}

The research was supported by the National Natural Science Foundation of China under Grant Nos. \#11471134, \#11771169, \#11871235, \#11571280, \#11771150, \#11301172, and \#11226170 and by the Hunan Provincial Natural Science Foundation of China under Grant No. \#2017JJ2105.

\section{References}

[1] L. Hsiao and T. T. Li, "Global smooth solution of cauchy problems for a class of quasilinear hyperbolic systems," Chinese Annals of Mathematics, vol. 4B, pp. 109-115, 1983. 
[2] T. T. Li and T. H. Qin, "Global smooth solutions for a class of quasilinear hyperbolic systems with dissipative terms," Chinese Annals of Mathematics, vol. 6B, pp. 199-210, 1985.

[3] T. Nishida, Nonlinear Hyperbolic Equations and Related Topics in Fluid Dynamics, Publications Mathematiques Docsay 78.02, Department de mathématique Paris-Sud, 1978.

[4] Y. S. Zheng, "Global smooth solution to the adiabatic gas dynamics system with dissipation terms," Chinese Annals of Mathematics, vol. 17A, pp. 155-162, 1996.

[5] L. Hsiao and T.-P. Liu, "Convergence to nonlinear diffusion waves for solutions of a system of hyperbolic conservation laws with damping," Communications in Mathematical Physics, vol. 143, no. 3, pp. 599-605, 1992.

[6] L. Hsiao and T.-P. Liu, "Nonlinear diffusive phenomena of nonlinear hyperbolic systems," Chinese Annals of Mathematics, vol. 14, pp. 465-480, 1993.

[7] K. Nishihara, "Convergence rates to nonlinear diffusion waves for solutions of system of hyperbolic conservation laws with damping," Journal of Differential Equations, vol. 131, no. 2, pp. 171-188, 1996.

[8] K. Nishihara, "Asymptotic behavior of solutions of quasilinear hyperbolic equations with linear damping," Journal of Differential Equations, vol. 137, no. 2, pp. 384-395, 1997.

[9] K. Nishihara, W. K. Wang, and T. Yang, " $L p$-convergence rate to nonlinear diffusion waves for $p$-system with damping," Journal of Differential Equations, vol. 161, pp. 191-218, 2000.

[10] H. J. Zhao, "Convergence to strong nonlinear diffusion waves for solutions of $p$-system with damping," Journal of Differential Equations, vol. 174, no. 1, pp. 200-236, 2001.

[11] H. J. Zhao, "Asymptotic behaviors of solutions of quasilinear hyperbolic equations with linear damping II," Journal of Differential Equations, vol. 167, no. 2, pp. 467-494, 2000.

[12] Z. Shao, "Global solutions with shock waves to the generalized riemann problem for a class of quasilinear hyperbolic systems of balance laws II," Mathematische Nachrichten, vol. 281, no. 6, pp. 879-902, 2008.

[13] Z. Shao, "Almost global existence of classical discontinuous solutions to genuinely nonlinear hyperbolic systems of conservation laws with small BV initial data," Journal of Differential Equations, vol. 254, no. 7, pp. 2803-2833, 2013.

[14] C. Dafermos, "A system of hyperbolic conservation laws with frictional damping," Zeitschrift für Angewandte Mathematik und Physik, vol. 46, pp. 294-307, 1995.

[15] L. Hsiao and S. Q. Tang, "Construction and qualitative behavior of the solution of the perturbed Riemann problem for the system of one-dimensional isentropic flow with damping," Journal of Differential Equations, vol. 123, pp. 480-503, 1995.

[16] H.-L. Li and K. Saxton, "Asymptotic behavior of solutions to quasilinear hyperbolic equations with nonlinear damping," Quarterly of Applied Mathematics, vol. 61, no. 2, pp. 295-313, 2003.

[17] C. K. Lin, C. T. Lin, and M. Mei, "Asymptotic behavior of solution to nonlinear damped $p$-system with boundary effect," International Journal of Numerical Analysis Model Series B, vol. 1, pp. 70-92, 2010.

[18] H. F. Ma and M. Mei, "Best asymptotic profile for nonlinear damped $p$-system with boundary effect," Journal of Differential Equations, vol. 249, pp. 446-484, 2010.
[19] M. Mei, "Best asymptotic profile for hyperbolic $p$-system with damping," SIAM Journal on Mathematical Analysis, vol. 42, pp. $1-23,2010$.

[20] M. Mei, "Nonlinear diffusion waves for hyperbolic $p$-system with nonlinear damping," Journal of Differential Equations, vol. 247, no. 4, pp. 1275-1296, 2009.

[21] C. J., "Convergence rates to nonlinear diffusion waves for weak entropy solutions to $p$-system with damping," Science in China Series A: Mathematics, vol. 46, no. 4, pp. 562-575, 2003.

[22] C. J. Zhu and M. N. Jiang, " $L^{p}$-decay rates to nonlinear diffusion waves for $p$-system with nonlinear damping," Science in China Series A, vol. 49, no. 6, pp. 721-739, 2006.

[23] T. P. Liu, A. Matsumura, and K. Nishihara, "Behavior of solutions for the burgers equations with boundary corresponding to the rarefaction waves," SIAM Journal on Mathematical Analysis, vol. 29, pp. 293-308, 1998.

[24] T. P. Liu and K. Nishihara, "Asymptotic behavior for scalar viscous conservation laws with boundary effect," Journal of Differential Equations, vol. 133, no. 2, pp. 296-320, 1997.

[25] T. P. Liu and S. H. Yu, "Propagation of stationary viscous Burgers shock under the effect of boundary," Archive for Rational Mechanics and Analysis, vol. 139, pp. 57-82, 1997.

[26] A. Matsumura and M. Mei, "Asymptotic toward viscous shock profile for solution of the viscous $p$-system with boundary effects," Archive for Rational Mechanics and Analysis, vol. 146, pp. 1-22, 1999.

[27] T. Pan, H. Liu, and K. Nishihara, "Asymptotic stability of the rarefaction wave of a one-dimensional model system for compressible viscous gas with boundary," Japan Journal of Industrial and Applied Mathematics, vol. 16, pp. 431-441, 1999.

[28] P. Marcati, M. Mei, and B. Rubino, "Optimal convergence rates to diffusion wave for solutions of the hyperbolic conservation laws with damping," Journal of Mathematical Fluid Mechanics, vol. 7, pp. 224-240, 2005.

[29] K. Nishihara and T. Yang, "Boundary effect on asymptotic behavior of solutions to the p-system with linear damping," Journal of Differential Equations, vol. 156, pp. 439-458, 1999.

[30] F. Hou, I. Witt, and H. C. Yin, "Global existence and blowup of smooth solutions of 3-D potential equations with timedependent damping," Pacific Journal of Mathematics, vol. 292, no. 2, pp. 389-426, 2018.

[31] X. H. Pan, "Global existence of solutions to 1-D Euler equations with time-dependent damping," Nonlinear Analysis, vol. 132, pp. 327-336, 2016.

[32] X. H. Pan, "Remarks on 1-D Euler equations with time-decayed damping," http://arxiv.org/abs/1510.08115.

[33] H. B. Cui, H. Y. Yin, J. S. Zhang, and C. J. Zhu, "Convergence to nonlinear diffusion waves for solutions of euler equations with time-depending damping," Journal of Differential Equations, vol. 264, no. 7, pp. 4564-4602, 2018.

[34] H. B. Cui, H. Y. Yin, C. J. Zhu, and L. M. Zhu, "Convergence to diffusion waves for solutions of euler equations with timedepending damping on quadrant," Science China Mathematics, vol. 62, pp. 33-62, 2019. 


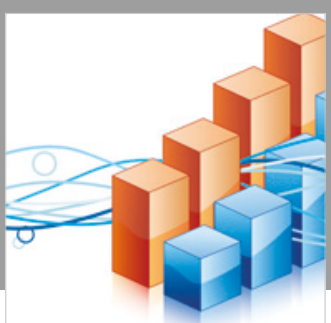

Advances in

Operations Research

\section{-n-m}
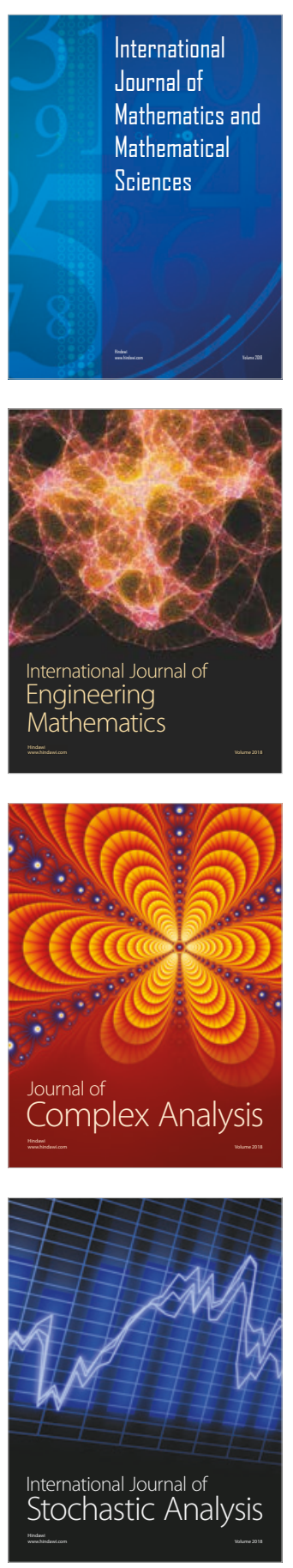
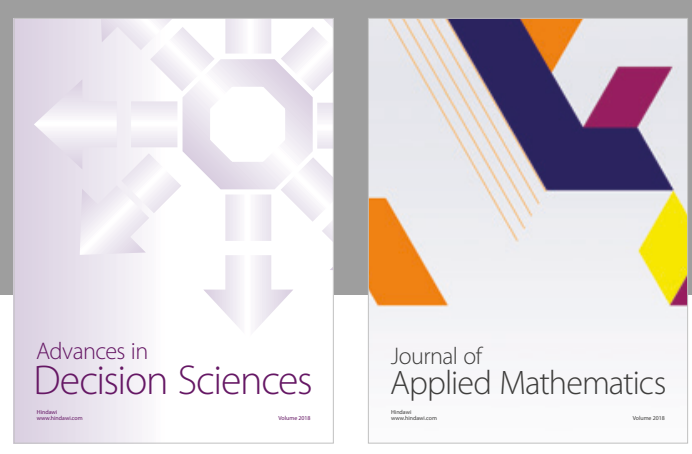

Journal of

Applied Mathematics
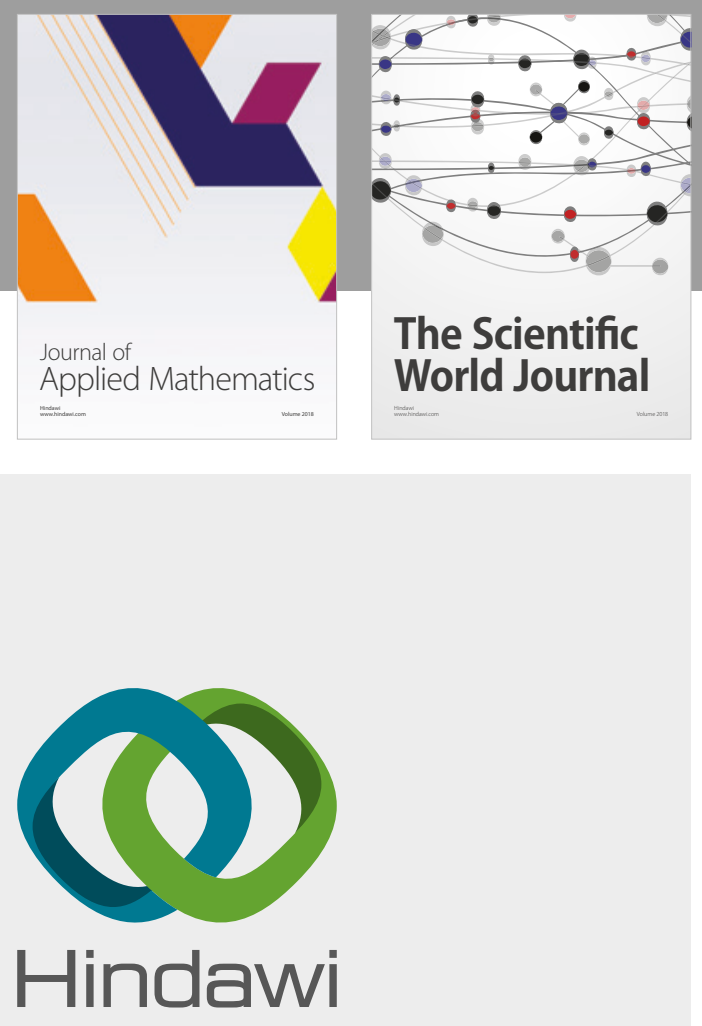

Submit your manuscripts at

www.hindawi.com

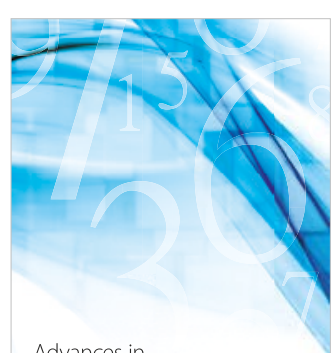

Advances in
Numerical Analysis
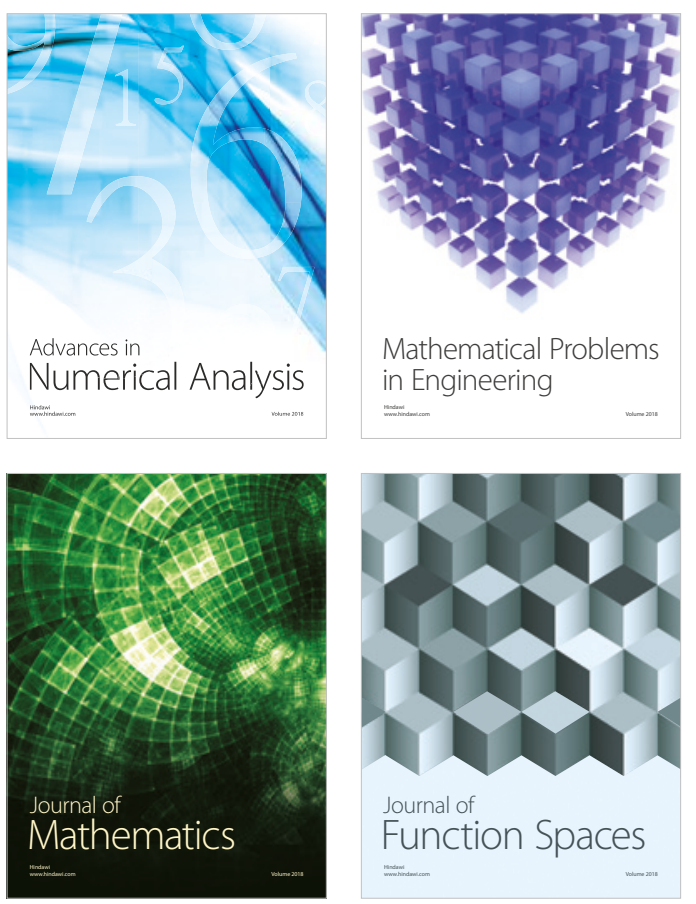

Mathematical Problems in Engineering

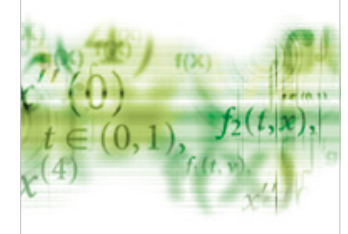

International Journal of

Differential Equations

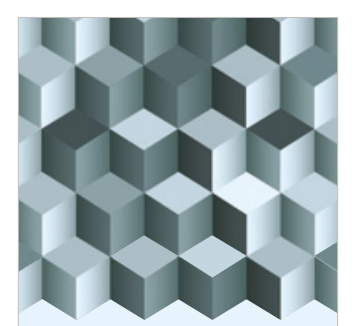

Journal of

Function Spaces
The Scientific

World Journal

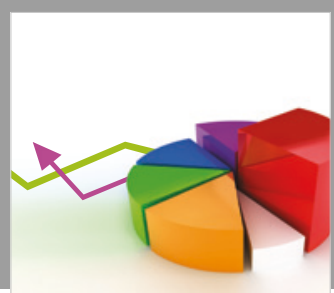

Journal of

Probability and Statistics
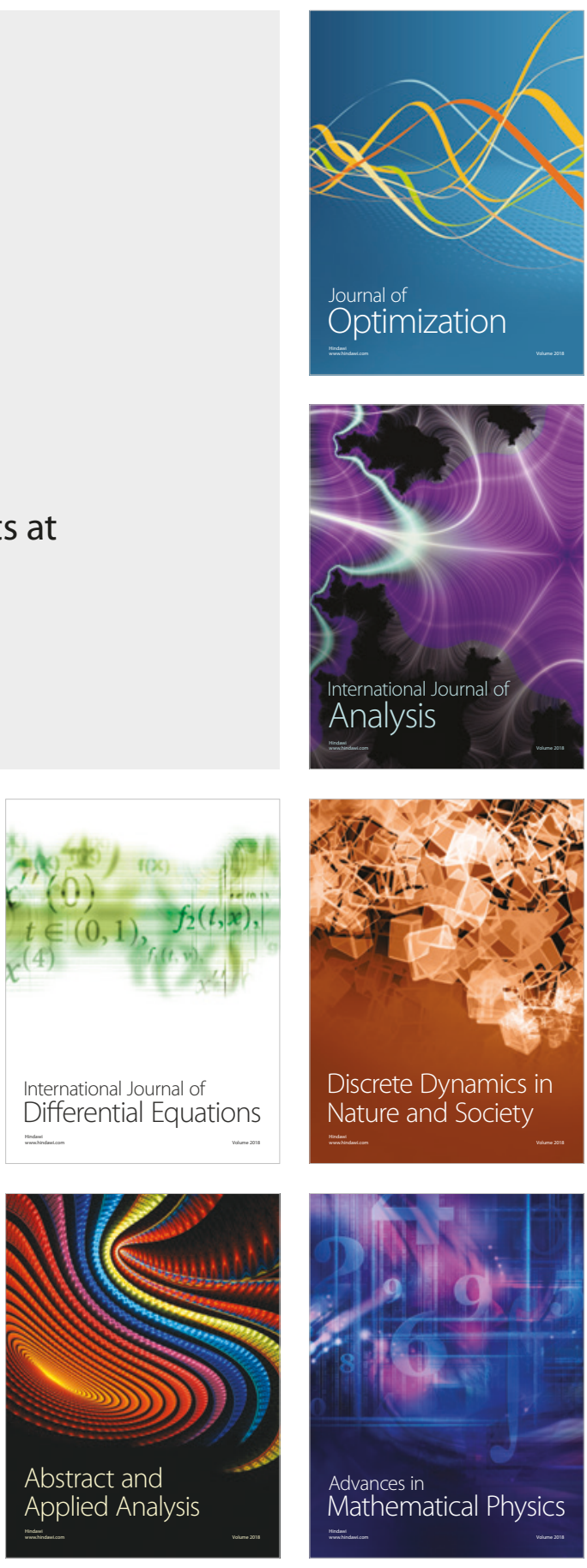